\title{
Mediennutzung und Medienkompetenz junger russischer Aussiedler in Nordrhein-Westfalen
}

\author{
Annett Heft / Torsten Maurer / Hans-Jürgen Weiß
}

Die Mediennutzung und Medienkompetenz von Menschen mit Migrationshintergrund ist Gegenstand einer breiten gesellschaftspolitischen und wissenschaftlichen Debatte. Neben positiven Erwartungen an die Bildungs-, Kommunikations- und Partizipationspotenziale insbesondere der digitalen Medien für junge Migranten stebt dabinter auch die Befürchtung, diese könnten die genannten Potenziale nicht in voller Bandbreite erschließen. Allerdings liegen zur Mediennutzung und Medienkompetenz junger Migranten in Deutschland bislang kaum repräsentative Basisdaten vor. Vor diesem Hintergrund präsentiert der Beitrag Ergebnisse einer repräsentativen Telefonbefragung zur Mediennutzung und Medienkompetenz von jungen russischen Aussiedlern im Alter von 12 bis 19 Jabren in Nordrhein-Westfalen und vergleicht diese mit Befunden für die gesamte Altersgruppe der in Deutschland lebenden 12- bis 19-Jährigen. Im Ergebnis verweist dieser Vergleich vor allem auf die Gemeinsamkeiten, die junge Menschen im Umgang mit den alten und den neuen Medien verbinden. Der Medienumgang der jungen russischen Aussiedler wird weniger durch ibren Migrationsstatus als vielmebr durch Lebensalter, Geschlecht und insbesondere Schulbildung bestimmt.

Schlagwörter: Mediennutzung, Medienkompetenz, Digital Divide, Migration

\section{Problemstellung}

Vor dem Hintergrund des ökonomisch und technologisch forcierten Wandels zur modernen Informations- und Wissensgesellschaft wird heute aus gesellschaftspolitischer Perspektive immer häufiger nach der „Medienkompetenz“ der Bevölkerung gefragt und erörtert, wie sie insbesondere bei Heranwachsenden als moderne Schlüsselqualifikation gefördert werden kann. In der Medienpädagogik gibt es schon seit den 1970er Jahren eine kontinuierliche Auseinandersetzung mit diesem Thema (Baacke 1973, 1999; Theunert 1999, 2008; Burkhardt 2001, Six/Gimmler 2007, Luca/Aufenanger 2007). Dementsprechend vielfältig sind auch die wissenschaftlichen Ansätze, die Kategorie der Medienkompetenz zu definieren (vgl. zusammenfassend Luca/Aufenanger 2007: 17-33). Theunert unterscheidet in diesem Zusammenhang vier Dimensionen der Medienkompetenz:

1. Wissen (Struktur- und Funktionswissen, instrumentelle Fertigkeiten),

2. Reflexion (Analyse, ästhetische Beurteilung, ethisch-soziale Wertung),

3. Orientierung (Positionierung, Einschätzung) und

4. Handlung (Gestaltung, kommunikativer Gebrauch, Partizipation).

Folgt man dieser Systematik, ist Medienkompetenz „die Fähigkeit, die Medien und Techniken, die gesellschaftliche Kommunikation unterstützen, steuern und tragen, erstens zu begreifen, zweitens sinnvoll damit umzugehen und drittens sie selbstbestimmt zu nutzen“ (Theunert 1999: 53).

Die aktuelle Medienkompetenz-Debatte ist zum einen mit positiven Erwartungen an die Bildungs- und Partizipationspotenziale der Medien verknüpft, die besonders auf die neuen digitalen Medien wie Computer, Internet, Handy etc. fokussiert sind. Zum anderen steht sie in einem engen Zusammenhang mit der These von einer digitalen Spaltung der Gesellschaft. Befürchtet wird, dass Bevölkerungsgruppen mit geringen Chancen auf 
Bildung, Beruf, politische Teilhabe und persönliche Entwicklung in Zukunft noch stärker benachteiligt sein könnten, wenn sie grundlegende Kenntnisse im Umgang mit den neuen digitalen Medienangeboten nicht erwerben können. Der ursprüngliche Ausgangspunkt der Digital Divide-These war die Beobachtung, dass verschiedene Bevölkerungsgruppen einen unterschiedlichen Zugang zu den neuen Kommunikationsmedien, insbesondere zum Internet, haben (Kubicek/Welling 2000). Mittlerweile geht es jedoch weniger um die Frage des Zugangs, vielmehr wird nun verstärkt die inhaltliche Ausrichtung des Mediengebrauchs - durchaus im Sinne des Medienkompetenzkonzepts von Theunert - in den Mittelpunkt der Debatte gerückt (van Dijk 2005, Zillien 2006). Die Rede vom „Second Digital Divide“ verdeutlicht, dass eher in der Intensität und vor allem in der Qualität der Nutzung dieser Medien wesentliche Unterschiede vermutet werden (Niesyto 2008, 2009).

Angesichts der Vielzahl der hierauf bezogenen Förderungsprojekte und einer relativ breit ausdifferenzierten Medienkompetenzforschung ist es erstaunlich, dass zur Mediennutzung und Medienkompetenz junger Migranten bisher kaum repräsentative Basisdaten vorliegen, obwohl hierauf bezogene Annahmen und Postulate in die medien- und gesellschaftspolitische Debatte eingehen (Medien- und Kommunikationsbericht der Bundesregierung 2008: 50). Der nachfolgende Beitrag knüpft an dieser Forschungslücke an. Sein empirischer Ausgangspunkt sind Daten einer Studie, die 2008 im Auftrag der Landesanstalt für Medien Nordrhein-Westfalen (LfM) zur „Mediennutzung Jugendlicher und junger Erwachsener mit Migrationshintergrund in Nordrhein-Westfalen“, so der Titel der Ausschreibung, durchgeführt wurde. ${ }^{1}$ In seiner allgemeinen Perspektive ist der Beitrag auf junge russische Aussiedler fokussiert, die konkreten empirischen Analysen sind auf die Altersgruppe der 12- bis 19-Jährigen begrenzt - nicht nur, weil es sich hierbei um eine relevante Zielgruppe für Maßnahmen zur Medienkompetenzförderung handelt, sondern auch, weil für diese Altersgruppe Vergleichsdaten zur Mediennutzung und Medienkompetenz in der deutschen Gesamtbevölkerung vorliegen (zuletzt: mpfs 2009b).

Das Ziel des Beitrags ist es, repräsentative Daten zur Nutzung klassischer Massenmedien sowie neuer digitaler Individualmedien durch 12- bis 19-jährige russische Aussiedler in Nordrhein-Westfalen zu präsentieren und diese Daten mit Befunden zur Mediennutzung und Medienkompetenz der gesamten Altersgruppe der in Deutschland lebenden 12- bis 19-Jährigen zu vergleichen. Aus der Perspektive der Medienkompetenzforschung liegt der Schwerpunkt der Analysen auf der Handlungsdimension (Mediengebrauch). Dieser Aspekt wird jedoch insbesondere bei den Analysen zur Nutzung der digitalen Individualmedien unter inhaltlichen und funktionalen Aspekten ausdifferenziert und durch Daten zur Wissens-, Reflexions- und Orientierungsfunktion ergänzt.

1 In der LfM-Studie wurde die Mediennutzung von Personen türkischer Herkunft und russischen Aussiedlern im Alter zwischen 12 und 29 Jahren in Nordrhein-Westfalen erfasst und in zwei Problemkontexten - der sozialen Integration und der Medienkompetenz junger Migranten analysiert. Hierzu wurden repräsentative Telefonbefragungen und Gruppendiskussionen durchgeführt (Trebbe et al. 2010; vgl. dazu auch den Beitrag von Paasch-Colberg/Trebbe in diesem Heft). 


\section{Junge russische Aussiedler in Deutschland}

Unter den rund 15,4 Millionen „Menschen mit Migrationshintergrund“2 Daten des Mikrozensus im Jahr 2007 in Deutschland lebten, befinden sich 4,9 Millionen Deutsche. Davon sind mindestens 2,8 Millionen als sog. „Aussiedler“ bzw. „Spätaussiedler"3 nach Deutschland eingereist (Statistisches Bundesamt 2009: 7). ${ }^{4}$

Während in der ersten Hälfte der 1990er Jahre die Zuzugsquoten dieser Migrantengruppe nach Deutschland bei über 200.000 Personen pro Jahr lagen, ist ihr Zuzug seitdem stetig zurückgegangen, 2007 betrug er nur noch 5.792 Personen (Migrationsbericht 2007: 46ff.). ${ }^{5}$ Vor ihrer Einreise nach Deutschland haben (Spät-)Aussiedler sowie deren Ehegatten und Nachkommen die Rechtsstellung eines „Deutschen ohne Staatsangehörigkeit“ („Statusdeutsche"). Werden sie als (Spät-)Aussiedler anerkannt, wird ihnen bei der Einreise nach Deutschland automatisch die deutsche Staatsbürgerschaft verliehen.

Seit 1993 kommen die Spätaussiedler fast ausschließlich aus den Gebieten bzw. Nachfolgestaaten der ehemaligen Sowjetunion. ${ }^{6}$ Nach Schneider sind „bis Ende 2004 [...] insgesamt rund 2,5 Millionen Menschen als Aussiedler, Spätaussiedler oder deren Angehörige aus der Sowjetunion bzw. ihren Nachfolgestaaten zugewandert" (Schneider 2005). Das ist der Personenkreis, den wir im Rahmen dieses Beitrags - in Übereinstim-

2 Die Bezeichnung „Menschen mit Migrationshintergrund“, die sich seit dem Mikrozensus 2005 durchgesetzt hat, umfasst nach der Definition des Statistischen Bundesamts „alle nach 1949 auf das heutige Gebiet der Bundesrepublik Deutschland Zugewanderten sowie alle in Deutschland geborenen Ausländer und alle in Deutschland als Deutsche Geborenen mit zumindest einem zugewanderten oder als Ausländer in Deutschland geborenen Elternteil“" (Statistisches Bundesamt 2009: 6). Aus Gründen der Lesbarkeit verwenden wir in diesem Beitrag den Begriff der „Migranten“ synonym zur korrekten Langformel der „Menschen mit Migrationshintergrund“.

3 „Aussiedler" sind nach $₫ 1$ Abs. 2 Nr. 3 Bundesvertriebenengesetz (BVFG) deutsche Staatsangehörige oder deutsche Volkszugehörige, die vor dem 1. Juli 1990 bis 31.12.1992 im Wege des Aufnahmeverfahrens die ehemals unter fremder Verwaltung stehenden deutschen Ostgebiete, Danzig, Estland, Lettland, Litauen, die ehemalige Sowjetunion, Polen, die Tschechoslowakei, Ungarn, Rumänien, Bulgarien, Jugoslawien, Albanien oder China verlassen haben (Bundesgesetzblatt Jahrgang 2007 Teil I Nr. 40: 1903). Die Rechtskategorie des „Aussiedlers“ nach $\$ 1$ Abs. 2 Nr. 3 BVFG wurde 1993 durch die neue Kategorie „Spätaussiedler“ abgelöst. „Spätaussiedler" sind in der Regel deutsche Volkszugehörige, die Benachteiligungen aufgrund deutscher Volkszugehörigkeit unterlagen und die Republiken der ehemaligen Sowjetunion $(\mathbb{} 4$ Abs. 1 BVFG) oder die in $\$ 1$ Abs. 2 Nr. 3 genannten Aussiedlungsgebiete ( $\$ 4$ Abs. 2 BVFG) nach dem 31.12.1992 im Wege des Aufnahmeverfahrens verlassen haben (Bundesgesetzblatt Jahrgang 2007 Teil I Nr. 40: 1904).

4 Nach Seifert (2008) unterschätzt der Mikrozensus allerdings die Aussiedlerquote in Deutschland.

5 Der Rückgang der Zuzugszahlen ist auch durch die Neuregelungen der Aufnahmevoraussetzungen durch das Kriegsfolgenbereinigungsgesetz 1992 und das Zuwanderungsgesetz 2005, welches den Nachweis von Grundkenntnissen der deutschen Sprache auch durch Ehegatten und Abkömmlinge erfordert, begründet.

6 Die große Mehrheit der Spätaussiedler stammt seitdem aus der Russischen Föderation oder Kasachstan. Bei Antragstellern aus den Nachfolgestaaten der ehemaligen Sowjetunion wird eine Benachteiligung aufgrund deutscher Volkszugehörigkeit weiterhin unterstellt ( $\mathbb{S}$ Abs. 1 BVFG). Antragsteller aus anderen Aussiedlungsgebieten müssen diese seit 1993 gesondert glaubhaft machen (Migrationsbericht 2007: 47-54). 
mung mit der einschlägigen Literatur ${ }^{7}$ - als „russische Aussiedler“ bezeichnen. Die Menschen, die diesem Bevölkerungssegment zuzuordnen sind, bilden neben den Menschen mit einem türkischen Migrationshintergrund die quantitativ umfangreichste Gruppe der in Deutschland lebenden Migranten.

Während die Integration von Aussiedlern in Deutschland bis in die 1980er Jahre relativ unproblematisch verlief, fällt die öffentliche Wahrnehmung und Einschätzung der Zuwanderung von Aussiedlern bereits seit Ende der 1980er Jahre deutlich negativer aus (Silbereisen et al. 1999a, Vogelgesang 2008). Dies dürfte nicht nur mit den veränderten Zuwanderungszahlen zusammenhängen, sondern auch mit der veränderten Zusammensetzung dieser Migrantengruppe. Zu Beginn der 1990er Jahre waren fast vier Fünftel der Zuwanderer selbst Spätaussiedler, die durch Sprache, Erziehung etc. in der deutschen Kultur verankert waren. Seit Ende der 1990er Jahre reisten dann deutlich mehr Ehegatten und Abkömmlinge von Spätaussiedlern ein (Migrationsbericht 2007: 49). Diese verfügten oft über schlechte deutsche Sprachkenntnisse und waren stärker in der russischen Kultur verwurzelt (Vogelgesang 2008, Strobl 2006). Die Sonderrolle der Aussiedler gegenüber anderen Migranten bleibt in diesen Fällen vor allem auf ihren rechtlichen Status beschränkt.

In der Literatur wird davon ausgegangen, dass besonders die jugendlichen Aussiedler der ersten Generation mit zusätzlichen Anpassungsschwierigkeiten konfrontiert sind, weil sich bei ihnen Probleme des Jugendalters und der Migration überlagern (Silbereisen et al. 1999b: 18). Die jugendlichen Aussiedler wurden teilweise sogar als „besondere Problemgruppe“ ausgemacht (Dietz 1997: 8). Auf den Jugendlichen würden die Hoffnungen der Eltern lasten, „als ,Deutsche unter Deutschen' in der Bundesrepublik Fuß zu fassen“ (ebd.), wovon diese aber aufgrund mangelnder Sprachkenntnisse und fehlender Orientierung in der westlichen Gesellschaft überfordert seien. So beschreiben Dietz und Roll auf Basis einer 1995/1996 durchgeführten Befragung unter jungen Aussiedlern den lebensgeschichtlichen Bruch, den die Ausreiseentscheidung für viele Jugendliche zur Folge hat (Dietz 1997, Dietz/Roll 1998). Die befragten Aussiedlerjugendlichen waren in ihrer ehemaligen Heimat sozial integriert, die meisten von ihnen standen der Ausreise mit gemischten Gefühlen gegenüber. Die Belastung wurde dann in Deutschland dadurch verstärkt, dass sie, auch aufgrund ihrer Sprachdefizite, eher als Russen wahrgenommen wurden und sich in Deutschland nicht willkommen fühlten. Eine Folge war, dass die jungen Aussiedler zunehmend unter sich blieben und wenige Kontakte zu einheimischen Jugendlichen hatten. Während laut Dietz und Roll noch die Mehrheit der Jugendlichen in den Ausreiseprozess mit eingebunden war, zeichnen jüngere Forschungen ein anderes Bild (vgl. Vogelgesang 2008). Demnach wollten viele junge Aussiedler nicht nach Deutschland kommen, sie wurden nicht gefragt und auch nicht darüber aufgeklärt, was sie in Deutschland erwartet - Faktoren, die als wenig integrationsförderlich eingeschätzt werden. So werden Aussiedlerjugendliche auch als "mitgenommene Generation“ (Schäfer 2002) bezeichnet, die „zuhause fremd“ bleibt (Ipsen-Peitzmeier/Kaiser 2006).

Diese knappen Ausführungen zeigen, dass junge russische Aussiedler trotz ihres privilegierten rechtlichen Status mit zahlreichen Problemlagen konfrontiert sind, die den Problemen anderer in Deutschland lebender junger Menschen mit Migrationshintergrund gleichen. Eines davon ist die Benachteiligung von Kindern und Jugendlichen mit

7 Sowohl in der wissenschaftlichen Literatur als auch umgangssprachlich ist der Begriff der „russischen Aussiedler“ oder auch der „Russlanddeutschen“ gebräuchlich (Ingenhorst 1997, IpsenPeitzmeier/Kaiser 2006). Er bezieht sich auf Personen aus dem Gebiet der ehemaligen Sowjetunion, wobei die Russische Föderation und Kasachstan die Hauptherkunftsgebiete sind (Migrationsbericht 2007: 53). 
Migrationshintergrund im deutschen Bildungssystem. Geißler/Weber-Menges (2008) zeigen, dass nach den Daten der bundesweiten Schulstatistik Jugendliche aus russischen Familien zwar deutlich besser abschneiden als zum Beispiel Jugendliche mit türkischem Migrationshintergrund. In Nordrhein-Westfalen nehmen russische Aussiedler im Hinblick auf ihre Schulabschlüsse aber trotzdem nur „eine mittlere Position zwischen Deutschen und Ausländern ein“ (Geißler/Weber-Menges 2008: 17). Dies lässt sich, so die Autoren, nicht nur auf schichtspezifische Ursachen zurückführen, sondern ist auch durch Integrationsprobleme begründet, die „bei der Wanderung in eine fremde Kultur mit einer anderen Verkehrs- und Unterrichtssprache, einem anderen Bildungssystem und teilweise anderen Werten und Normen entstehen" (ebd.: 18).

\section{Forschungsüberblick: Mediennutzung und Medienkompetenz junger russischer Aussiedler in Deutschland}

Auch wenn die Problemstellungen der Medienkompetenzforschung weit über die Ermittlung von Basisdaten zur Mediennutzung potenzieller Zielgruppen der Medienkompetenzförderung hinausreichen, muss man sich in Wissenschaft und Praxis der grundlegenden Frage stellen, was man tatsächlich über den Medienumgang in der Bevölkerung bzw. in einzelnen Bevölkerungsgruppen und Handlungsfeldern weiß. Bezogen auf die deutsche Gesellschaft als Ganze ist das auf den ersten Blick kein Problem. In der angewandten Mediaforschung werden durch bevölkerungsrepräsentative Studien der Arbeitsgemeinschaft Media-Analyse e.V. (AGMA) und der Arbeitsgemeinschaft Fernsehforschung (AGF) kontinuierlich Basisdaten zur Reichweite und Nutzung der „klassischen“ Medien (Zeitungen, Zeitschriften, Hörfunk, Fernsehen) ermittelt. Dazu kommt die in längeren Zeitabständen im Auftrag von ARD und ZDF durchgeführte Studie „Massenkommunikation“, die auch die Internetnutzung erfasst (Ridder/Engel 2005). Außerdem gibt es zu den neueren Problemstellungen der Medienkompetenzforschung, die besonders auf den Umgang von Kindern und Jugendlichen mit digitalen Funktionsbzw. Individualmedien ausgerichtet sind, seit den 1990er Jahren zwei jährlich durchgeführte Repräsentativerhebungen des Medienpädagogischen Forschungsverbunds Südwest (mpfs): die sog. KIM-Studie („Kinder und Medien, Computer und Internet“), die sich auf 6- bis 13-Jährige, und die sog. JIM-Studie („Jugend, Information, (Multi-)Media“), die sich auf 12- bis 19-Jährige bezieht (zuletzt: mpfs 2009a, 2009b).

Diese Studien schließen aus ihren Stichprobenerhebungen in Deutschland lebende Menschen mit Migrationshintergrund nicht systematisch aus, aber auch nicht systematisch ein. Repräsentative Informationen über ihre Mediennutzung und Medienkompetenz sind auf dieser Basis nicht zu erhalten. ${ }^{8}$ Damit stellt sich zwangsläufig die Frage, ob es in Deutschland eine mit diesen Studien vergleichbare Forschung gibt, in der (a) die Mediennutzung sowie (b) spezielle Indikatoren zur Medienkompetenz von (c) (jungen) Menschen mit Migrationshintergrund auf repräsentativer Basis erfasst werden.

Der erste Teil der Frage ist im Grundsatz positiv zu beantworten. Jedoch war diese Forschung, trotz einer Forschungstradition, die in die Anfänge der 1980er Jahre zurückreicht, bisher eher eklektisch und außerdem stark auf Menschen türkischer Her-

8 Die Datenerhebung zum weiter unten erwähnten (N)Onliner Atlas 2008 stellt insofern eine Ausnahme dar, als in ihr der Migrationshintergrund der Befragten selbst bzw. der ihrer Eltern ermittelt wurde. Die Grundgesamtheit dieser Studie bildet die deutschsprachige Wohnbevölkerung ab 14 Jahren mit Festnetzanschluss im Haushalt (Initiative D21e.V. 2008). 
kunft fokussiert (Trebbe 2009: 60f.). ${ }^{9}$ Allerdings wurde durch eine 2007 von ARD und ZDF durchgeführte Studie eine Wende zu mehr Kontinuität und Breite eingeleitet, sofern diese tatsächlich - wie geplant - in regelmäßigen Abständen wiederholt wird (Simon 2007). ${ }^{10}$ Im Rahmen dieser Studie wurde unter anderem auch die Mediennutzung russischer Aussiedler ab 14 Jahren repräsentativ erfasst. Ansonsten ist im Bezug auf russische Aussiedler nur noch eine 1999 durchgeführte Repräsentativerhebung zur Mediennutzung von in Niedersachsen lebenden russischen Aussiedlern ab 18 Jahren zu erwähnen (Pfetsch/Weiß 2000, Pfetsch/Trebbe 2003, Ivanova 2009).

Weitgehend negativ fällt dagegen die Beantwortung des zweiten und dritten Teils der oben gestellten Frage aus. Die Untersuchungsperspektive der zuletzt genannten Repräsentativerhebungen ist vorwiegend auf die Erfassung der Reichweite und Nutzung der „klassischen“ Massenmedien Zeitung, Zeitschrift, Radio und vor allem Fernsehen ausgerichtet. Von den neuen digitalen Individual- bzw. Funktionsmedien wird nur das Internet berücksichtigt und das auch nur ganz allgemein im Hinblick auf Nutzungsdauer und Nutzungshäufigkeit. Aspekte der Medienkompetenz spielen in dieser Forschung ebenso wenig eine Rolle, wie sie auf junge Menschen mit Migrationshintergrund fokussiert ist.

Immerhin rückt die Nutzung des Internets durch Migranten immer stärker in den Fokus der Forschung. Das lässt sich zum Beispiel an einer aktuellen Sonderauswertung des (N)Onliner Atlas 2008 ablesen, die sich mit grundlegenden Kennwerten zur Nutzung bzw. Nicht-Nutzung des Internets durch sechs Teilpopulationen mit Migrationshintergrund ${ }^{11}$ befasst, darunter Befragte aus dem Gebiet der ehemaligen Sowjetunion (Initiative D21e.V. 2008). Auch die Forschungen von Kissau (2008) sowie Kissau/Hunger (2009) zu den Integrations- und Partizipationspotenzialen des Internets für postsowjetische bzw. jüdische Migranten und ein aktuelles Forschungsprojekt von WeberMenges, welches russische Aussiedler berücksichtigt (vgl. dazu den Hinweis in Geißler/ Weber-Menges 2009), bieten neue Befunde zur Internetnutzung von Migranten. Aber auch für diese Studien gilt die Einschränkung, dass sie nicht auf junge Menschen mit Migrationshintergrund fokussiert sind.

Zusammenfassend ist zu konstatieren, dass es in Deutschland bisher keine explizit auf junge Migranten bezogene Mediennutzungs- und Medienkompetenzforschung gibt, der repräsentative Basisinformationen über den Umgang dieser Population mit den „alten“ und den „neuen“ Medien zu entnehmen sind. Was wir daher über den Medienumgang junger russischer Aussiedler in Deutschland wissen, stammt aus zwei unterschiedlichen wissenschaftlichen Quellenbereichen:

Auf der einen Seite gibt es breit angelegte Medienkompetenzstudien, zu deren Untersuchungspopulation de facto junge Migranten und damit auch junge Aussiedler zählen. Typisch hierfür sind Schülerstudien, wie z. B. die Untersuchung der Forschergruppe

9 Als Ausnahme ist hier vor allem die Medienforschung des Westdeutschen Rundfunks zu nennen, die an die erste „Ausländerstudie“ von ARD und ZDF anknüpfte und sich mehrfach mit der Mediennutzung mehrerer Migrantengruppen in Nordrhein-Westfalen beschäftigte (Eckhardt 2000, Windgasse 2007).

10 Befragt wurden Personen mit türkischem, ex-jugoslawischem, polnischem, italienischem und griechischem Migrationshintergrund sowie (Spät-)Aussiedler aus dem Bereich der ehemaligen Sowjetunion.

11 Die Stichprobe umfasst Personen mit türkischem, ex-jugoslawischem, polnischem, rumänischem und italienischem Migrationshintergrund sowie Zuwanderer aus dem Gebiet der ehemaligen Sowjetunion (Nennungen $>100$ ). Daneben gehen weitere Herkunftsländer mit Nennungen $<100$ ein. 
um Klaus Peter Treumann zum „Bielefelder Medienkompetenzmodell“ (Treumann et al. 2007) ${ }^{12}$ oder eine Analyse zum „Medienhandeln in Hauptschulmilieus“ (Wagner 2008), bei der zwei Drittel der befragten Schülerinnen und Schüler einen Migrationshintergrund aufweisen. ${ }^{13}$ Obwohl in diesen Studien eine große Zahl junger Migranten erfasst wird, ist bei der Generalisierung ihrer Ergebnisse Vorsicht geboten. Dagegen spricht vor allem die Klumpenbildung bei der Auswahl der Schulen, Schulklassen und Schüler. Wenn dann noch der Schwerpunkt der Erhebung auf „Problemschulen in Problemregionen“ liegt (Wagner 2008), verbietet sich jede Verallgemeinerung der Befunde.

Auf der anderen Seite wurde in den letzten Jahren eine nicht geringe Anzahl qualitativer Studien durchgeführt, die explizit den Medienumgang junger Aussiedler aus kompetenzbezogener Perspektive und mit Blick auf neue digitale Medien beleuchten in der Regel jedoch unter Verzicht auf repräsentative Verfahren der Datenerhebung (so z. B. Eggert 2006, Treibel 2006, Düvel 2009, Hepp et al. 2009). Daraus ergibt sich auch hier ein Vorbehalt gegen die Generalisierbarkeit der Ergebnisse dieser Forschung. Unabhängig davon geben wir im Folgenden einen kurzen Überblick über diejenigen Befunde, die sich auf den Medienumgang junger russischer Aussiedler beziehen und dabei auf Aspekte der Medienkompetenz fokussieren oder einen Vergleich zu deutschen Jugendlichen ohne Migrationshintergrund herstellen. ${ }^{14}$

\section{1 „Klassische“ Massenmedien}

Dietz und Roll weisen darauf hin, dass der Fernsehkonsum bei jungen russischen Aussiedlern ausgeprägter sei als bei deutschen Jugendlichen ohne Migrationshintergrund (Dietz/Roll 1998: 115). Eine mögliche Ursache könnte eine mangelnde soziale Einbindung in einen Freundeskreis in den ersten Jahren nach der Zuwanderung sein. Außerdem werde deutsches Fernsehen gerade in der Anfangszeit genutzt, um Deutsch zu lernen. Russisches Fernsehen und russische Filme gelten demgegenüber als ein Element, das die Familien im neuen Lebenskontext verbindet, und auch als Möglichkeit der Rückbindung an die Aussiedlungsgebiete (Eggert 2006, Elias/Lemish 2008). Über die Nutzung des Radios und die Lesepräferenzen junger Aussiedler liegen kaum Befunde vor, zur Zeitungsnutzung gibt es Hinweise darauf, dass zum Beispiel kostenlose Stadtzeitungen regelmäßig gelesen würden (Eggert 2006). ${ }^{15}$

\section{2 „Neue“Funktions- bzw. Individualmedien}

Die Nutzung von Computer und Internet, sei es im instrumentellen, performativen oder kommunikativen Sinne, setzt zunächst einmal die Ausstattung mit bzw. einen Zugang

12 Ein Teilkriterium für die Definition der Grundgesamtheit war, dass die befragten Jugendlichen Deutsch als Muttersprache beherrschten. Daneben wurde ihre Nationalität erfasst und ermittelt, ob sie schon immer in Deutschland lebten oder aus dem Ausland zugezogen sind.

13 In der Studie wurden Hauptschüler aus 20 Haupt- und Gesamtschulen in sozialen Brennpunkten bzw. strukturschwachen Gebieten von Großstädten befragt. Bezogen auf den Migrationshintergrund wurde für Vater und Mutter gefragt, ob sie aus Deutschland oder woanders her kommen.

14 Den erstgenannten, breit angelegten Medienkompetenzstudien sind keine explizit auf junge Aussiedler bezogenen Befunde zu entnehmen.

15 Sekundäranalysen z. B. der von ARD und ZDF 2007 durchgeführten Studie (Simon 2007, Oehmichen 2007) für junge Aussiedler (z. B. im Alter zwischen 14 und 19 Jahren) wären hier durchaus hilfreich. 
zu diesen Medien voraus. Hierzu gibt es in der Literatur Anzeichen dafür, dass der Zugang zu Computer und Internet für jugendliche Aussiedler - in Abhängigkeit von Geschlecht, Bildung und Aufenthaltsdauer in Deutschland - erschwert sein könnte (Treibel 2006, Eggert 2006). Diesem Mangel werde jedoch begegnet, indem Computer und Internet bei Freunden, Verwandten und in Internetcafés genutzt werden. Die Unterstützungsleistung der Familie wird in diesem Zusammenhang von Treibel allerdings ambivalent eingeschätzt. Die Mehrheit der von ihr befragten Hauptschüler und -schülerinnen ${ }^{16}$ könne nicht auf ihre Familien als Kompetenzträger zurückgreifen, zudem seien sie für die Relevanz des Kompetenzerwerbs im Bereich der neuen Medien nicht sensibilisiert. Gefragt nach ihrem Interesse an Wissens- und Kompetenzzuwachs bezüglich des Internets sahen die Jugendlichen keinen Bedarf und hielten ihre Kompetenz für ausreichend - eine Einschätzung, die von der Autorin der Studie nicht geteilt wird (Treibel 2006: 223f.).

Die neuen Medien scheinen aber auch neue Freiräume zu schaffen sowie Kommunikations- und Beteiligungschancen zu eröffnen, die sich die Jugendlichen zunutze machen. Eine zentrale Rolle der neuen Medien liegt demnach in deren Brückenfunktion (Treibel 2006, Eggert 2006). Das Internet werde nicht nur zum Recherchieren und zum Spielen genutzt, sondern auch zur Kontaktaufnahme mit der Familie und Freunden im Herkunftsland. Die Beteiligung an russischsprachigen Chats oder der Austausch mit anderen Zuwanderern in Deutschland und weltweit per Internet ist demzufolge bei den in diesen Studien befragten Jugendlichen besonders beliebt. Die Chatbekanntschaften beschränken sich dabei nicht notwendigerweise auf den Chat an sich, sondern sie erfahren eine Fortsetzung im „realen“ Leben (Eggert 2006, Vogelgesang 2008).

Auf die Beteiligungschancen, die insbesondere digitale Medien durch die Möglichkeit zu kommunikativen Mehrfachvernetzungen eröffnen könnten, hat auch Düvel hingewiesen (Düvel 2009: 266). Anhand einer Fallstudie zeigt sie, dass je nach Migrationsbiografie und kultureller Selbstverortung verschiedene Medien für verschiedene kommunikative Vernetzungen eingesetzt werden: SMS zum Beispiel für die lokale Kommunikation mit Freunden, Internetkommunikation via Skype nach Russland. Digitale Medien ermöglichen so die Inklusion in räumlich getrennte soziale und kulturelle Kontexte (Hepp et al. 2009).

In diesem Zusammenhang ist die sprachliche Flexibilität der Jugendlichen hervorzuheben. Die Kommunikation finde je nach Bedarf sowohl in Deutsch als auch in der Herkunftssprache statt (Treibel 2006), was sich zum Teil in russischsprachigen Benutzeroberflächen auf den Computern äußere (Eggert 2006). Russische Internetseiten könnten z. B. helfen, Sprachbarrieren zu überwinden. Eine „russische Netzkultur“ macht auch Vogelgesang (2008: 194) aus und betont, dass das Internet den jungen Aussiedlern insbesondere als Medium der Sichtbarmachung und Selbstverortung ethnischer Zugehörigkeit diene. Die Nutzung der kommunikativen und partizipativen Möglichkeiten des Internets könnte auf diese Weise zu Identitätsbildungsprozessen bei jungen russischen Aussiedlern beitragen.

Die Nutzung von Mobiltelefonen durch junge russische Aussiedler und diesbezügliche Medienkompetenzaspekte waren bislang kaum Forschungsgegenstand (Treibel 2006, Hepp et al. 2009). Problematisiert wird dagegen die Nutzung von Computer- und Onlinespielen (Eggert 2006: 249, 254; Fromme et al. 2000). Anscheinend gibt es Gründe

16 Dabei handelt es sich um russische Aussiedler und türkischstämmige Jugendliche im Alter von durchschnittlich 15 Jahren. 
für die Vermutung, dass bei männlichen Heranwachsenden mit Aussiedlerhintergrund actionhaltige und kampfbetonte Angebote überdurchschnittlich beliebt sein könnten.

\section{Die Methode der Telefonbefragung junger russischer Aussiedler in Nordrhein- Westfalen}

In Kapitel 5 und 6 dieses Beitrags werden Daten zur Mediennutzung und Medienkompetenz von jungen russischen Aussiedlern, die in Nordrhein-Westfalen leben, präsentiert und mit Befunden verglichen, die für die deutsche Gesamtbevölkerung in diesem Alterssegment repräsentativ sind. Es handelt sich dabei um eine Sonderauswertung einer Studie der Landesanstalt für Medien Nordrhein-Westfalen (LfM), die sich insgesamt aus breiterer Perspektive mit der Mediennutzung von jungen Migranten in NordrheinWestfalen befasst (Trebbe et al. 2010). Auf die Methode der Gesamtstudie wird im Folgenden nur so weit eingegangen, wie es zum Verständnis der Ergebnisse der Sonderauswertung erforderlich ist. ${ }^{17}$

\subsection{Grundgesamtheit, Stichprobenverfahren und Untersuchungsinstrument}

Im Mittelpunkt der LfM-Studie stehen zwei Populationen junger Migranten in Nordrhein-Westfalen: Personen türkischer Herkunft und russische Aussiedler. Der repräsentative Teil der Datenerhebungen stützt sich auf Telefonumfragen (CATI-Verfahren).

Die Grundgesamtheit für die Befragung der russischen Aussiedler wurde in folgender Weise definiert: „Russische Aussiedler zwischen 12 und 29 Jahren in privaten Haushalten mit Telefoneintrag in Nordrhein-Westfalen “. ${ }^{18}$ Die Stichprobenziehung erfolgte in zwei Stufen: auf der ersten, regional geschichteten Stufe (Telefonhaushalte) nach dem namensbasierten Onomastik-Verfahren (Humpert/Schneiderheinze 2000), auf der zweiten Stufe (Zielpersonen) nach der Last-Birthday-Methode. ${ }^{19}$

Die Bruttostichprobe umfasste 2.225, die Nettostichprobe 1.664 Telefonhaushalte. In 1.159 Haushalten konnte ein Interview geführt werden (Ausschöpfungsquote 70 \%), davon $n=303$ Voll-Interviews mit Personen, die das Alterskriterium 12 bis 29 Jahre

17 Vgl. dazu auch die Methodendokumentation auf der Homepage der LfM (http://lfm-nrw.de/ forschung/schriftreihe).

18 Die Telefonumfragen wurden von ENIGMA GfK Medien- und Marketingforschung GmbH, Wiesbaden, durchgeführt.

19 Das Onomastik-Verfahren hat sich als Methode zur Ziehung von Telefonstichproben in Migrantenpopulationen durchgesetzt und wurde auch in der ARD/ZDF-Studie 2007 eingesetzt (dort allerdings noch nicht im Bezug auf russische Aussiedler, vgl. Simon 2007). Dieses Verfahren nutzt Erkenntnisse der Namensforschung, um die sprachliche Herkunft von Namen zu ermitteln und die Trefferwahrscheinlichkeit bei der Listenauswahl für die telefonische Befragung von Migranten zu verbessern. Auf Basis der Erfassung von Vor- und Nachnamen sowie Informationen zur Migrationsgeschichte werden den Listeneinträgen Treffererwartungen unterschiedlicher Güte zugewiesen. Für die vorliegende Aussiedler-Stichprobe wurden Adressen mit der Trefferwahrscheinlichkeit 1 (Einträge mit einer eindeutig plausiblen Zuordnung, z. B. Vorname und Nachname einer Nationalität) und 2 (Einträge mit zwei plausiblen Zuordnungen, z. B. Vorname und Nachname zweier Nationalitäten) ausgewählt. Zu Beginn des Interviews wurde geprüft, inwieweit und wie viele Personen mit dem Migrationshintergrund „russische (Spät-)Aussiedler“ im Haushalt wohnen. In nur 12 Prozent der für diese Stichprobe gezogenen Haushalte lebte keine Person mit dem erwarteten Migrationshintergrund. Diese Quote ist nur geringfügig höher als bei der Ziehung der Haushalte mit (erwartetem) türkischem Migrationshintergrund (9\%). 
erfüllten. Die Stichprobe wurde auf der Basis der Daten des Mikrozensus 2007 für Nordrhein-Westfalen nach Alter und Geschlecht gewichtet. Dabei zeigt sich, dass - gemessen am Mikrozensus - jüngere Befragte in den Originaldaten der Stichprobe relativ stark überrepräsentiert sind. ${ }^{20}$ In den gewichteten Daten ist die Altersgruppe der 12bis 19-jährigen russischen Aussiedler, auf die sich die nachfolgenden Analysen beziehen, mit $\mathrm{n}=132$ Fällen vertreten.

Die beiden Teilpopulationen der LfM-Studie wurden mit einem inhaltlich identischen Untersuchungsinstrument befragt. Die zentrale Zielvorgabe für die Entwicklung des Fragebogens bestand darin, dass zu den drei Analysekomplexen: (a) Mediennutzung, (b) Medienkompetenz und (c) soziale Integration Daten erhoben werden sollten, die nicht nur im Hinblick auf die Befunde zu den beiden Teilpopulationen, sondern auch mit den Ergebnissen einschlägiger Repräsentativerhebungen in der deutschen Gesamtbevölkerung sowie mit anderen Migrantenstudien kompatibel sind. Bezogen auf die in diesem Beitrag behandelte Problemstellung rückt damit die in Kapitel 3 erwähnte JIMStudie in den Vordergrund, in der insbesondere die Nutzung und Einschätzung der neuen digitalen Medien in der Altersgruppe der 12- bis 19-Jährigen in jährlich wiederholten Telefonumfragen ermittelt wird. Ein Großteil der auf diesen Themenkomplex bezogenen Fragen der JIM-Studie wurde in die LfM-Studie übernommen.

Hieraus erklärt sich nun auch die Begrenzung der nachfolgenden Analysen auf die in der LfM-Studie befragten 12- bis 19-jährigen russischen Aussiedler: Die für diese Migrantengruppe ermittelten Daten sollen, soweit möglich und sinnvoll, im Vergleich zu den Daten betrachtet werden, die im Rahmen der JIM-Studie 2008 (mpfs 2008) für die gesamte Altersgruppe der in Deutschland lebenden 12- bis 19-Jährigen ermittelt worden sind. ${ }^{21}$

Für die Telefonbefragung wurden ausschließlich zweisprachige Interviewerinnen eingesetzt. Die Befragten konnten selbst wählen, in welcher Sprache sie sich interviewen lassen wollten. Bei den russischen Aussiedlern im Alter zwischen 12 und 19 Jahren entschieden sich 93 Prozent für Deutsch als Interviewsprache. Von ihnen sprachen nach Einschätzung der Interviewerinnen drei Viertel „sehr gut“ Deutsch.

Ein besonderes komparatistisches Problem in diesem Forschungsfeld ergibt sich allerdings aus unterschiedlichen Definitionen, Messungen und Ausweisungen von „Mediennutzung“. In den neueren Repräsentativerhebungen zur Mediennutzung von Migranten hat sich - sieht man von der aufwendigen Tagesablauferhebung der ARD/ZDFStudie 2007 ab - die Kategorie der „Stammnutzer“ eingebürgert und bewährt. Das sind diejenigen Personen, die auf die Frage, „an wie vielen von den sieben Tagen einer normalen Woche“ sie ein bestimmtes Medium bzw. ein bestimmtes Medienangebot nutzen, mindestens vier Tage angeben. Diese Abfrage wurde daher auch in der LfM-Studie verwendet. In der JIM-Studie wird bei der Abfrage der Mediennutzung zwischen „täglich“, „mehrmals pro Woche“, „einmal pro Woche“, „einmal alle 14 Tage“, „einmal pro Monat“ und „seltener“ unterschieden. Zur Ausweisung von Nutzungswerten werden in der Regel die Angaben „täglich“ und „mehrmals pro Woche“ zusammengefasst.

20 Vgl. dazu allerdings die Argumente von Seifert (2008) gegen die Repräsentativität des Mikrozensus für die Gruppe der Aussiedler in Nordrhein-Westfalen.

21 Dabei wiegt die Chance, die Migrantendaten in dieser Altersgruppe relativieren zu können, die methodischen Probleme dieses Vergleichs weitgehend auf. Problematisch ist zum einen der unterschiedliche regionale Kontext des Vergleichs (russische Aussiedler/Nordrhein-Westfalen vs. Gesamtbevölkerung/Bundesrepublik Deutschland), zum anderen die mangelnde Trennschärfe der Daten für die Gesamtbevölkerung bezüglich des Migrationshintergrunds. 
Hier beginnen nun die Probleme des Studienvergleichs. Die Nutzungskategorie der JIM-Studie ist mit Sicherheit breiter als die der LfM-Studie. In der Konsequenz werden im Folgenden in den Vergleichstabellen nicht nur die (im engeren Kontext der Migrationsforschung relevanten) Stammnutzerdaten der LfM-Studie ausgewiesen, sondern zusätzlich auch die auf mindestens drei Nutzungstage pro Woche bezogenen Untersuchungsergebnisse. Der Vergleich wird damit komplexer, die Gefahr von Artefakten (im Sinne der methodischen Erzeugung von Differenz) dadurch jedoch reduziert.

\subsection{Die Stichprobe}

\section{Soziodemografie}

Aufgrund der Gewichtung der Daten spiegeln sich in der internen Altersstruktur der LfM-Stichprobe und im jeweiligen Anteil von Jungen und Mädchen die Gegebenheiten wider, die für die in Nordrhein-Westfalen lebenden Aussiedler durch den Mikrozensus 2007 repräsentativ ermittelt worden sind. Im Vergleich zur JIM-Stichprobe 2008 für die Gleichaltrigen in der Gesamtbevölkerung sind in der Aussiedlerstichprobe die 12bis 15 -Jährigen ( 35 vs. 46 \%) unter- und die 18- bis 19-Jährigen überrepräsentiert (40 vs. $28 \%$ ).

Der weitaus größte Teil der 12- bis 19-Jährigen geht noch zur Schule (84 \%), jeder Zehnte befindet sich in einer Ausbildung. In diesem Punkt entspricht die LfM-Stichprobe ziemlich genau der Zusammensetzung der JIM-Stichprobe 2008. Relativ große Unterschiede betreffen jedoch den Schultyp. Bei den jungen Aussiedlern, die noch zur Schule gehen, ist der Anteil der Haupt- und Realschüler im Vergleich zu den Gleichaltrigen in der Gesamtbevölkerung mit 25 bzw. 49 Prozent deutlich überrepräsentiert. Der Anteil der Gymnasiasten ist dagegen stark unterrepräsentiert (26\%). ${ }^{22}$ Andererseits ist ein Großteil der jungen russischen Aussiedler durchaus aufstiegsorientiert: Nur 11 Prozent der Schüler wollen sich mit einem Hauptschulabschluss zufrieden geben, 34 Prozent streben die Mittlere Reife an und 45 Prozent wollen Fachabitur oder Abitur machen. Das entspricht fast den „realen“ Bildungswerten der Gleichaltrigen in der Gesamtbevölkerung.

Fast alle der befragten 12- bis 19-jährigen Aussiedler leben noch zu Hause bei ihren Eltern, drei Viertel zusammen mit Geschwistern. Die Hälfte von ihnen lebt in VierPersonen-Haushalten, ein Viertel in Haushalten mit fünf und mehr Personen.

\section{Migrationshintergrund}

Sehr viele junge russische Aussiedler haben eigene Migrationserfahrungen: Vier von fünf Befragten sind aus den Nachfolgestaaten der ehemaligen Sowjetunion ausgereist, nur ein Fünftel ist in Deutschland geboren. Allerdings leben über 70 Prozent der Befragten schon länger als fünf Jahre in Deutschland, über 40 Prozent sogar länger als zehn Jahre. Die deutsche Staatsbürgerschaft ist in dieser Migrantengruppe - wie in Abschnitt 2 erläutert - der Regelfall. Nur 5 Prozent der Befragten sind keine deutschen Staatsbürger.

Nicht nur unter Integrationsgesichtspunkten, sondern auch im Hinblick auf die Optionen, deutsch- und/oder russischsprachige Medienangebote $\mathrm{zu}$ nutzen, sind die Sprachkompetenzen und Sprachgewohnheiten der jungen russischen Aussiedler von Interesse. Deutsch zu verstehen, ist für die meisten 12- bis 19-jährigen Aussiedler, die in

22 Vgl. dazu die Ausführungen in Kap. 2 zur Bildungsproblematik der russischen Aussiedler in Nordrhein-Westfalen (Geißler/Weber-Menges 2008: 17). 
der LfM-Studie befragt wurden, kein Problem. Und nur 3 Prozent bezeichnen ihre Fähigkeit, Deutsch zu schreiben, als gering, 80 Prozent dagegen als gut bzw. sehr gut. Mit den Russischkenntnissen sieht es anders aus. Zwar verstehen drei Viertel der Befragten nach eigener Aussage Russisch gut und sehr gut. Gut und sehr gut Russisch sprechen kann jedoch nur gut die Hälfte der Befragten, Russisch lesen noch ein Viertel und Russisch schreiben sogar nur 15 Prozent.

Erwartungsgemäß sind die Aussiedlerfamilien der Ort, an dem das Russische im Alltag der Jugendlichen am stärksten tradiert wird. Ein Drittel der Befragten gibt an, dass bei ihnen Zuhause nur bzw. meistens Russisch gesprochen wird, bei einem weiteren Drittel wird „genauso viel Russisch wie Deutsch“ gesprochen. Die Zweisprachigkeit spielt außerdem noch in der Freizeit, im Umgang mit Freunden und Bekannten eine gewisse Rolle (28\%). Aber selbst dort dominiert das Deutsche (66\%).

Etwa die Hälfte der jungen Aussiedler hat in gleichem Umfang Umgang mit deutschen und russischen Freunden. Bei 30 Prozent überwiegt der deutsche, bei 20 Prozent der russische Anteil der Freunde. In Wohnvierteln mit vielen russischen Freunden in der Nähe leben gut 40 Prozent der jungen russischen Aussiedler.

Das Interesse am aktuellen öffentlichen Geschehen hält sich auch in diesem Segment der Jugendkultur in Grenzen. Als „sehr interessiert“ am aktuellen Geschehen in Deutschland bezeichnen sich 39 Prozent der Befragten. Bezogen auf das aktuelle Geschehen in Russland sind es 22 Prozent.

\section{Mediennutzung und Medienkompetenz 12- bis 19-jähriger russischer Aussiedler in Nordrhein-Westfalen}

Die Verfügbarkeit von Medien schafft die strukturellen Voraussetzungen für das alltägliche Medienhandeln. Daher wird zunächst ein knapper Überblick über die persönliche Medienausstattung der jungen russischen Aussiedler sowie der Haushalte gegeben, in denen sie leben (5.1). Die Befunde der LfM-Studie zum Medienhandeln der jungen russischen Aussiedler werden in zwei Abschnitten zusammengefasst. Zunächst wird die Nutzung von Massenmedien (unter Einschluss allgemeiner Rahmendaten zur Internetnutzung) skizziert (5.2), ehe dann relativ ausführlich auf die Nutzung der neuen digitalen Individualmedien eingegangen wird (5.3).

Aus der Perspektive der Medienkompetenzforschung liegt der Schwerpunkt dieses Abschnitts eindeutig auf der Ebene der Handlungsdimension. Primär hierzu werden empirisch belastbare Basisinformationen zusammengetragen. Bezogen auf die Nutzung der neuen digitalen Medien geht es jedoch um mehr als „äußere“ Rahmendaten: Hier werden die Informationen über den Mediengebrauch der jungen russischen Aussiedler unter inhaltlichen und funktionalen Gesichtspunkten ausdifferenziert sowie durch Befunde zur reflexiven Einordnung und Bewertung dieser Medien durch die Befragten ergänzt.

\subsection{Medienausstattung}

In nahezu allen Haushalten, in denen die jungen russischen Aussiedler leben, steht mindestens ein Fernsehgerät; mehr als die Hälfte der Befragten hat ein eigenes Gerät (vgl. Tab. 1). Drei Viertel der Haushalte verfügen über digitale Empfangsmöglichkeiten. Dieser Wert entspricht der Quote der Ja-Antworten auf die Frage, ob russische Fernsehprogramme empfangen werden können. Auch das Radio gehört noch weitgehend zur Standard-Medienausstattung der Aussiedlerhaushalte (74\%), die Tageszeitung jedoch nicht. Nur in gut einem Viertel der Haushalte wird eine Tageszeitung abonniert. Deut- 
lich stärker verbreitet als die beiden letztgenannten „klassischen“ Massenmedien sind die neuen digitalen „Individual- bzw. Funktionsmedien“: Handy, PC, MP3-Player/iPod etc. Außerdem verfügen fast alle Aussiedlerhaushalte über einen Zugang zum Internet.

\section{Tabelle 1: Medienausstattung}

Mehrfachnennung, in Prozent (gew.)

\begin{tabular}{lcccc}
\hline & \multicolumn{2}{c}{ Haushalte } & \multicolumn{2}{c}{ Jugendliche } \\
\hline & $\begin{array}{c}\text { LfM-Studie 08 } \\
\text { nw=132 }\end{array}$ & $\begin{array}{c}\text { JIM-Studie 08 } \\
\text { nw=1.208 }\end{array}$ & $\begin{array}{c}\text { LfM-Studie 08 } \\
\text { nw=132 }\end{array}$ & $\begin{array}{c}\text { JIM-Studie 08 } \\
\text { nw=1.208 }\end{array}$ \\
\hline Zeitungsabonnement & 28 & 59 & -1 & -1 \\
Radiogerät & 74 & -2 & 34 & 77 \\
Fernsehgerät & 97 & 98 & 58 & 61 \\
Internetzugang & 93 & 96 & 48 & 51 \\
Computer & 96 & 99 & 65 & 71 \\
MP3-Player/iPod & 88 & 93 & 81 & 86 \\
CD-Player & 83 & 94 & 56 & 76 \\
Feste Spielkonsole & 55 & 65 & 37 & 45 \\
Mobile Spielkonsole & 40 & 53 & 25 & 41 \\
Handy & 98 & 99 & 93 & 95 \\
\hline
\end{tabular}

1 Zeitungsabonnements wurden in beiden Studien nicht als individuelles Ausstattungsmerkmal erfasst.

2 Die Ausstattung der Haushalte mit Radiogeräten wurde in der JIM-Studie 2008 nicht erhoben.

Sieht man von Radio und Tagespresse ab, leben damit junge russische Aussiedler im Alter zwischen 12 und 19 Jahren weitgehend in derselben privaten Medienumgebung wie ihre Altersgenossen in der deutschen Gesamtbevölkerung. Betrachtet man ihre eigene, individuelle Medienausstattung, gilt im Prinzip dasselbe. Die Rangfolge der Medien ist - mit Ausnahme des Radios - in beiden Populationen gleich. Im Bezug auf PC-, MP3-Playerund Spielkonsolen-Besitz liegen die Werte für die jungen Aussiedler etwas unter dem Durchschnitt für alle 12- bis 19-Jährigen. Diese Differenz betrifft aber nicht den Internetzugang und Handy-Besitz.

\subsection{Massenmediennutzung ${ }^{23}$}

Dass sich die Medienausstattung der Aussiedlerhaushalte im alltäglichen Umgang der jungen russischen Aussiedler mit Massenmedien widerspiegelt, ist selbstverständlich. Das Internet wird an dieser Stelle in die Betrachtung einbezogen, da es grundsätzlich auch als Massenmedium (bzw. als Zugangsweg zu massenmedialen Angeboten) genutzt werden kann.

Im Gesamtspektrum des Mediennutzungsverhaltens der 12- bis 19-jährigen russischen Aussiedler ist das Fernsehen ganz offensichtlich das Massenmedium Nummer 1. Es wird im Vergleich zu den anderen Massenmedien am häufigsten, regelmäßigsten und längsten genutzt. Wie ihre Altersgenossen in der Gesamtbevölkerung sehen ca. 90 Pro-

23 Zur Problematik des Vergleichs zwischen den Mediennutzungsdaten der LfM- und der JIMStudie vgl. die Ausführungen in Abschnitt 4.1. Die im Text referierten Daten der LfM-Studie beziehen sich, wenn nicht anders ausgewiesen, auf eine Nutzungsfrequenz von mindestens drei Tagen pro Woche. 
Tabelle 2: Massenmediennutzung

Mebrfachnennung, in Prozent (gew.)

\begin{tabular}{lccc}
\hline & $\begin{array}{c}\text { LfM-Studie 08 } \\
\mathrm{nw}=132\end{array}$ & $\begin{array}{c}\text { LfM-Studie } 08^{2} \\
\mathrm{nw}=132\end{array}$ & $\begin{array}{c}\text { JIM-Studie 083 } \\
\mathrm{nw}=1.208\end{array}$ \\
\hline Fernsehen & 86 & 91 & 89 \\
Internet & 73 & 80 & 84 \\
Radio & 34 & 39 & 72 \\
Zeitung & 12 & 19 & 43 \\
\hline
\end{tabular}

1 Personen, die angeben, das Medium an mindestens 4 Tagen einer normalen Woche zu nutzen.

2 Personen, die angeben, das Medium an mindestens 3 Tagen einer normalen Woche zu nutzen.

3 Täglich/Mehrmals pro Woche.

zent der jungen russischen Aussiedler zumindest mehrfach in der Woche fern (vgl. Tab. 2) - die meisten von ihnen täglich.

Nicht ganz so hoch sind die mit der Fernsehnutzung vergleichbaren Pauschalwerte für die regelmäßige Internetnutzung ( $80 \%$ ). Bezogen auf die Nutzung des Internets als Massenmedium gibt die LfM-Studie folgende Hinweise: Etwa ein Viertel der Befragten gibt an, mehrmals pro Woche „aktuelle Nachrichten“ abzurufen, und etwa ein Drittel schaut mehrmals pro Woche Filme und Videos im Internet an. Die Angaben zum Empfang von Radio- und Fernsehprogrammen via Internet liegen weit unter 10 Prozent (vgl. auch Tab. 6). Das heißt zusammengenommen, dass die Internetnutzung der 12- bis 19jährigen Aussiedler zum weitaus größten Teil der Individualkommunikation und nicht dem Gebrauch von Massenmedien zuzuordnen ist.

Während sich die jungen russischen Aussiedler im quantitativen Umfang der Fernseh- und Internetnutzung kaum von Gleichaltrigen in der Gesamtbevölkerung unterscheiden, ist dies im Bezug auf das Radiohören und Zeitungslesen durchaus der Fall. Man findet unter ihnen nur sehr wenige regelmäßige Zeitungsleser, lediglich rund 20 Prozent greifen mehrmals pro Woche zur Tageszeitung. Und auch die Zahl der regelmäßigen Radiohörer ist mit ca. 40 Prozent relativ gering. Dass die Tagespresse in Deutschland große Schwierigkeiten hat, junge Leser zu erreichen, ist bekannt. Dennoch wird sie in der Gesamtbevölkerung von den 12- bis 19-Jährigen im Durchschnitt stärker genutzt als von den gleichaltrigen russischen Aussiedlern: In der JIM-Studie 2008 werden mehr als 40 Prozent Befragte ausgewiesen, die zumindest mehrmals pro Woche Zeitung lesen. Dasselbe gilt für das Radiohören, das in dieser Altersgruppe sogar relativ stark verbreitet ist $(72 \%)$.

Russischsprachige Medienangebote spielen im Alltag der jungen russischen Aussiedler insgesamt eine vergleichsweise geringe Rolle. Weniger als 20 Prozent der Befragten schauen sich täglich russische Fernsehprogramme an und nur 15 Prozent von ihnen rufen täglich russische Internetseiten auf. Die russischsprachige Internetnutzung nimmt somit insgesamt keinen so hohen Stellenwert ein, wie auf Basis der Literatur zu erwarten war. Auch die Nutzung von Radio und Zeitung in russischer Sprache ist weitgehend zu vernachlässigen. Bezogen auf die vier Medien Fernsehen, Radio, Zeitung und Internet kombinieren 28 Prozent der jungen Aussiedler die deutschsprachige mit der russischen Mediennutzung, d. h. sie nutzen an mindestens vier Tagen einer Woche deutsch- und russischsprachige Medienangebote parallel.

Aus einem etwas anderen Blickwinkel haben die Russischkenntnisse eine deutlich größere Bedeutung für die Massenmediennutzung der jungen russischen Aussiedler - es geht dabei um das instrumentelle Informationsverhalten: 53 Prozent der jungen russi- 
schen Aussiedler nutzen Medien in russischer Sprache, um sich über aktuelle Ereignisse in ihrem Herkunftsland zu informieren, und auch für Informationen über aktuelle Ereignisse in Deutschland greifen noch 43 Prozent auf russischsprachige Medien als Informationsquellen zurück. Dass dies in der Regel zusätzlich zur Nutzung deutscher Medien erfolgt, kann diesen Befund nicht schmälern.

\subsection{Individualmediennutzung}

Handy, Computer und Internet bestimmen den digitalen Medienalltag der 12- bis 19jährigen russischen Aussiedler. Vier Fünftel der Befragten gehen regelmäßig mit diesen Medien um (vgl. Tab. 3). Diese Werte unterscheiden sich nicht signifikant von den Nutzungswerten, die im Rahmen der JIM-Studie 2008 für die Gleichaltrigen in der deutschen Gesamtbevölkerung festgestellt worden sind. Dasselbe gilt auch für den deutlich selteneren Gebrauch digitaler Spielkonsolen (LfM-Studie: 22 Prozent; JIM-Studie: 26 \%).

\section{Tabelle 3: Individualmediennutzung Mebrfachnennung, in Prozent (gew.)}

\begin{tabular}{lccc}
\hline & $\begin{array}{c}\text { LfM-Studie 08 } \\
\mathrm{nw}=132\end{array}$ & $\begin{array}{c}\text { LfM-Studie 08 } \\
\mathrm{nw}=132\end{array}$ & $\begin{array}{c}\text { JIM-Studie 08 } \\
\text { nw=1.208 }\end{array}$ \\
\hline Computer & 71 & 83 & 89 \\
Internet & 73 & 80 & 84 \\
Handy & 75 & 80 & 84 \\
Spielkonsole (fest oder mobil) & 16 & 22 & 26 \\
\hline
\end{tabular}

1 Personen, die angeben, das Medium an mindestens 4 Tagen einer normalen Woche zu nutzen. 2 Personen, die angeben, das Medium an mindestens 3 Tagen einer normalen Woche zu nutzen. 3 Täglich/Mehrmals pro Woche.

\section{PC und Internet}

Computer werden von über 80 Prozent der jungen russischen Aussiedler vor allem zu Hause genutzt. Angesichts der guten Geräteversorgung der Haushalte, in denen die Befragten leben, ist das auch nicht verwunderlich. Wie die allgemeinen Daten zur Mediennutzung zeigen, besteht die Hauptfunktion der PCs und Notebooks vor allem darin, eine Verbindung zum Internet herzustellen. Andere Möglichkeiten der Computernutzung treten demgegenüber deutlich in den Hintergrund. Dies gilt allerdings für alle Jugendlichen dieses Alters: Nur sechs weitere Tätigkeiten am PC werden von mindestens 10 Prozent der 12- bis 19-Jährigen in Deutschland mehrmals pro Woche und öfter ausgeführt (vgl. Tab. 4).

Bei den jungen russischen Aussiedlern steht in diesem Zusammenhang das Spielen am PC an erster Stelle $(29 \%)^{24}$, an zweiter Stelle folgt ein instrumenteller Umgang mit dem PC für Bildungs- und berufliche Zwecke (24\%). Bei den Jugendlichen in der Gesamtbevölkerung ist das genau anders herum. Sie spielen zwar ebenso häufig wie die russischen Aussiedler am PC (30\%), jedoch hat bei ihnen die PC-Nutzung für Schule, Ausbildung und Beruf einen deutlich höheren Stellenwert (37\%). Auch bei der Textverarbeitung und produktiv-gestalterischen Tätigkeiten wie der Zusammenstellung von

24 Bei diesem Wert wird nicht zwischen Offline- und Online-Spielen unterschieden. 
Tabelle 4: Computernutzung

Mebrfachnennung, in Prozent (gew.)

\begin{tabular}{lccc}
\hline & $\begin{array}{c}\text { LfM-Studie 08 } \\
\mathrm{nw}=132\end{array}$ & $\begin{array}{c}\text { LfM-Studie 08 } \\
\mathrm{nw}=132\end{array}$ & $\begin{array}{c}\text { JIM-Studie 083 } \\
\mathrm{nw}=1.208\end{array}$ \\
\hline Spiele & 22 & 29 & 30 \\
Schule, Ausbildung, Beruf & 14 & 24 & 37 \\
Textverarbeitung & 12 & 17 & 27 \\
Bilder/Filme bearbeiten & 6 & 15 & 13 \\
DVD ansehen & 6 & 11 & 10 \\
Musik zusammenstellen & 4 & 9 & 18 \\
\hline
\end{tabular}

1 Personen, die angeben, das Medium an mindestens 4 Tagen einer normalen Woche zu nutzen. 2 Personen, die angeben, das Medium an mindestens 3 Tagen einer normalen Woche zu nutzen. 3 Täglich/Mehrmals pro Woche.

Musik liegen die jungen Aussiedler unter dem Durchschnitt - nicht jedoch im Umgang mit visuellem Material (Bilder/Filme bearbeiten, DVDs ansehen).

Bei der Beantwortung von Einstellungsfragen zur PC-Nutzung steht auch bei den meisten russischen Aussiedlern der instrumentelle Nutzen von Computern für Schule, Ausbildung und Beruf im Vordergrund. 95 Prozent der Aussiedler und 92 Prozent der JIM-Befragten bezeichnen den Computer in dieser Hinsicht als nützlich. 70 Prozent der Aussiedler und 78 Prozent der Jugendlichen der Gesamtbevölkerung stimmen dem Statement zu, dass Computer wichtig sind, um einen Beruf zu finden. ${ }^{25}$ Allerdings betonen die jungen Aussiedler mehr als andere Gleichaltrige den "Spaßfaktor" des Lernens am PC ( $81 \%$ vs. $70 \%) .{ }^{26}$ Gleichwohl ist das Interesse der jungen Aussiedler, sich häufiger mit dem Computer zu beschäftigen, geringer als bei den 12- bis 19-Jährigen der Gesamtbevölkerung: 27 Prozent der Aussiedler signalisieren ein solches Interesse, bei den JIM-Befragten sind es 38 Prozent. ${ }^{27}$ Auf ihr spezifisches soziales Umfeld verweist die Tatsache, dass immerhin 37 Prozent angeben, ihre Eltern würden nichts von Computern halten. Dieser Wert liegt deutlich über dem Durchschnitt für die Gleichaltrigen in der deutschen Bevölkerung ( $25 \%)$.

Wenn man zunächst untersucht, wofür die jungen Aussiedler das Internet nach eigener Aussage am häufigsten nutzen, stehen vor allem die Kommunikationsfunktionen des Internets an erster Stelle, aber auch Informations- und Unterhaltungsfunktionen sind von zentraler Bedeutung (vgl. Tab. 5). Die Dienste, die von zwei Drittel der Befragten mindestens drei Tage pro Woche genutzt werden, sind Messenger, ICQ etc. sowie Suchmaschinen. Mehr als die Hälfte von ihnen nutzt das Internet als Musikmedium und zur Partizipation an Online-Communities. Und knapp die Hälfte geht ins Internet, um zu surfen und sich per E-Mail und in Chat-Foren auszutauschen. Systematisiert man die Modalitäten der Internetnutzung und vergleicht auf dieser Basis die jungen russischen Aussiedler mit Gleichaltrigen in der deutschen Gesamtbevölkerung, findet man in der Migrantenstichprobe nur sehr wenige Besonderheiten (vgl. Tab. 6).

25 Vgl. JIM-Studie 2007 (2008 nicht erfasst).

2681 Prozent der Aussiedler und 70 Prozent der JIM-Befragten stimmen dem Statement „Es macht Spaß, mit dem Computer zu lernen oder zu arbeiten." voll und ganz oder weitgehend zu.

27 Zustimmung voll und ganz/weitgehend zum Statement „Eigentlich würde ich mich ganz gerne häufiger mit Computern beschäftigen." 
Tabelle 5: Hauptfunktionen des Internets in Prozent (gew.)

\begin{tabular}{ccll}
\hline Rang & $\begin{array}{c}\text { LfM-Studie } 08^{1} \\
\text { nw=132 }\end{array}$ & Tätigkeit & Funktion \\
\hline 1 & 68 & Messenger, ICQ etc. & Kommunikation \\
2 & 67 & Suchmaschinen & Information \\
3 & 58 & Musik/Sounds hören & Unterhaltung \\
4 & 55 & Online-Communities & Kommunikation \\
5 & 45 & Surfen & Information \\
6 & 43 & E-Mail & Kommunikation \\
7 & 43 & Chat & Kommunikation \\
8 & 36 & Informationen für Schule/Beruf & Information \\
9 & 32 & Filme/Videos ansehen & Unterhaltung \\
10 & 29 & Private Informationen & Information \\
\hline
\end{tabular}

1 Personen, die angeben, das Medium an mindestens 3 Tagen einer normalen Woche zu nutzen.

Kommunikation. Messenger, Online-Communities und E-Mails werden von allen 12- bis 19-Jährigen in Deutschland als die drei wichtigsten Kommunikationsmedien genutzt. In dieser Hinsicht ist - zumindest auf der Ebene der Nutzungshäufigkeiten - kein besonderes Kommunikationsverhalten der jungen russischen Aussiedler festzustellen, auch nicht, was den in der Literatur hervorgehobenen Stellenwert der Partizipation in Online-Communities betrifft.

Dagegen ist der Stellenwert der Chats bei den jungen russischen Aussiedlern deutlich höher als bei den Gleichaltrigen in der Gesamtbevölkerung (43 \% vs. 28 \%), was mit den referierten Studien übereinstimmt. Vor dem Hintergrund der geringen Anteile an russischsprachiger Internetnutzung (vgl. Abschnitt 5.2) dürfte jedoch nur ein Teil der Chatnutzung eine Brückenfunktion zum Herkunftsland haben. Dafür spricht unter anderem auch der Sachverhalt, dass drei Viertel der jungen Migranten dem Statement „Über das Internet kann man gut neue Leute kennen lernen“ zustimmen (dieser Wert ist deutlich höher als bei den Gleichaltrigen in der Gesamtbevölkerung).

Information. In der Nutzung des Internets als Informationsquelle unterscheiden sich die 12- bis 19-jährigen Aussiedler kaum von anderen Jugendlichen in ihrem Alter. Die Rangfolge der am stärksten genutzten Angebote und Dienste ist gleich. Nur das Interesse an privaten und Veranstaltungsinformationen ist etwas geringer als im Durchschnitt.

Unterhaltung. Hier ist vor allem interessant, dass sich - entgegen einer weit verbreiteten Annahme - das Spielverhalten der jungen Aussiedler im Internet quantitativ nicht vom durchschnittlichen Verhalten anderer Gleichaltriger unterscheidet. Aus der Unterhaltungsperspektive viel wichtiger für sie ist die Möglichkeit, im Internet Musik zu hören. Und hierin unterscheiden sie sich tatsächlich von den anderen (58 \% vs. $46 \%$ ).

Gestaltung. In der insgesamt in dieser Altersgruppe nicht besonders weit verbreiteten gestalterischen Komponente findet man dann doch noch ein geringfügiges „Defizit“ der Internetnutzung in der untersuchten Gruppe jugendlicher Migranten. Während im Durchschnitt 13 Prozent der Jugendlichen eine eigene Homepage gestalten, ist dies bei fast keinem der befragten russischen Aussiedler der Fall.

Vor diesem Hintergrund ist es nicht überraschend, dass sich die Ansichten der 12bis 19-jährigen russischen Aussiedler zum Internet im Grunde genommen nicht von denen anderer Jugendlicher in ihrem Alter unterscheiden. Dasselbe gilt für ihre Antworten auf Wissensfragen über das Internet. Der Nützlichkeit des Internets für Schule 
Tabelle 6: Internetnutzung

Mehrfachnennung, in Prozent (gew.)

\begin{tabular}{|c|c|c|c|}
\hline & $\begin{array}{c}\text { LfM-Studie } 08^{1} \\
n w=132\end{array}$ & $\begin{array}{c}\text { LfM-Studie } 08^{2} \\
n w=132\end{array}$ & $\begin{array}{c}\text { JIM-Studie } 08^{3} \\
\text { nw }=1.208\end{array}$ \\
\hline \multicolumn{4}{|l|}{ Kommunikation } \\
\hline Messenger, ICQ etc. & 61 & 68 & 71 \\
\hline Online-Communities & 46 & 55 & 55 \\
\hline E-Mail & 33 & 43 & 47 \\
\hline Chat & 41 & 43 & 28 \\
\hline \multicolumn{4}{|l|}{ Information } \\
\hline Suchmaschinen & 57 & 67 & 71 \\
\hline Surfen & 37 & 45 & 42 \\
\hline Informationen für Schule/Beruf & 22 & 36 & 38 \\
\hline Private Informationen & 21 & 29 & 38 \\
\hline Aktuelle Nachrichten & 23 & 27 & 32 \\
\hline Newsgroups lesen & 17 & 21 & 22 \\
\hline Veranstaltungsinformationen & 4 & 5 & 13 \\
\hline \multicolumn{4}{|l|}{ Unterbaltung } \\
\hline Musik/Sounds hören & 45 & 58 & 46 \\
\hline Filme/Videos ansehen & 23 & 32 & 26 \\
\hline Multi-User-Spiele & 12 & 16 & 19 \\
\hline Online-Spiele allein & 10 & 14 & 15 \\
\hline \multicolumn{4}{|l|}{ Gestaltung } \\
\hline Newsgroup schreiben & 7 & 13 & 13 \\
\hline Foto/Video einstellen & 4 & 6 & 10 \\
\hline Eigene Homepage & - & 2 & 13 \\
\hline
\end{tabular}

1 Personen, die angeben, das Medium an mindestens 4 Tagen einer normalen Woche zu nutzen. 2 Personen, die angeben, das Medium an mindestens 3 Tagen einer normalen Woche zu nutzen. 3 Täglich/Mehrmals pro Woche.

und Beruf stimmen 95 Prozent der Aussiedler und 93 Prozent der Befragten der JIMStudie $^{28}$ „voll und ganz“ oder „weitgehend“ zu. Die Selbsteinschätzung des eigenen Wissens über erlaubte und unerlaubte Angebote ist in beiden Populationen ähnlich hoch: 79 Prozent der jungen Aussiedler und 75 Prozent der JIM-Befragten stimmen dem Statement „Wenn ich das Internet nutze, weiß ich genau, was erlaubt ist und was nicht" voll und ganz bzw. weitgehend zu.

Dass dieses Selbstbewusstsein nicht immer gerechtfertigt ist und daher durchaus Potenzial für weitere Information und Aufklärung in dieser Altersgruppe über Internetstrukturen und -inhalte besteht, zeigen die Zustimmungswerte von 30 (russische Aussiedler) bzw. 28 Prozent (JIM-Befragte) zu dem Statement „Was im Internet steht, hat vorher jemand auf die Richtigkeit überprüft".

28 Vgl. JIM-Studie 2007 (2008 nicht erfasst). 


\section{Mobiltelefon}

Zwei Drittel der 12- bis 19-jährigen Aussiedler nutzen ihr Mobiltelefon mit einer Prepaid-Karte, ein Viertel hat einen Handy-Vertrag. Die Durchschnittskosten für das mobile Telefonieren liegen monatlich bei 18 Euro, der Eigenanteil beträgt 10 Euro. Diese Rahmendaten sind weitgehend mit den Durchschnittswerten für diese Altersgruppe in der deutschen Gesamtbevölkerung identisch.

\section{Tabelle 7: Handynutzung}

Mebrfachnennung, in Prozent (gew.)

\begin{tabular}{|c|c|c|c|}
\hline & $\begin{array}{c}\text { LfM-Studie } 08^{1} \\
n w=132\end{array}$ & $\begin{array}{c}\text { LfM-Studie } 08^{2} \\
n w=132\end{array}$ & $\begin{array}{c}\text { JIM-Studie } 08^{3} \\
n w=1.208\end{array}$ \\
\hline Angerufen werden & 63 & 75 & 71 \\
\hline SMS bekommen & 54 & 64 & 76 \\
\hline Musik mp3 hören & 59 & 62 & 43 \\
\hline Jemanden anrufen & 48 & 59 & 57 \\
\hline SMS schicken & 43 & 55 & 69 \\
\hline Fotos/Filme machen & 22 & 33 & 37 \\
\hline Fotos/Filme senden Bluetooth & 15 & 24 & 23 \\
\hline MP3 senden Bluetooth & 14 & 23 & 23 \\
\hline Handyspiele & 6 & 11 & 10 \\
\hline Handy-Radio & - & 1 & 11 \\
\hline
\end{tabular}

1 Personen, die angeben, das Medium an mindestens 4 Tagen einer normalen Woche zu nutzen. 2 Personen, die angeben, das Medium an mindestens 3 Tagen einer normalen Woche zu nutzen. 3 Täglich/Mehrmals pro Woche.

In der Grundtendenz gilt das auch für die verschiedenen Möglichkeiten, dieses mobile Multifunktionsgerät zu nutzen (vgl. Tab. 7). Bei allen Jugendlichen in dieser Altersgruppe stehen zwei Funktionsbereiche - die kommunikativen Primärfunktionen (Telefonieren, SMS) sowie unterhaltende Sekundärfunktionen (Musik, Fotos/Filme, Spiele) - im Vordergrund. Alle weiteren Funktionen, insbesondere in Verbindung mit dem Internet, sind relativ irrelevant. Am wichtigsten sind tatsächlich die kommunikativen Funktionen des Handys. Interessanterweise rangieren dabei die passiven (angerufen werden, SMS bekommen) vor den aktiven Funktionen (SMS schicken, jemanden anrufen). Dabei scheinen die jungen russischen Aussiedler häufiger zu telefonieren und seltener die SMS-Funktion nutzen als dies in dieser Altersgruppe im Durchschnitt der Fall ist.

Am auffälligsten ist jedoch der hohe Stellenwert, den bei ihnen die Verwendung des Handys als MP3-Player hat. Die Differenz zu den Durchschnittswerten für die Gleichaltrigen in der Gesamtbevölkerung ist enorm (62\% vs. $43 \%)$. Das gilt umgekehrt allerdings in einer sehr viel schwächeren Ausprägung - für die Nutzung des Handys zum Hören von Radioprogrammen. Diese Funktion wird von einer kleinen Gruppe ihrer Altersgenossen genutzt (ca. 10 Prozent mindestens mehrmals pro Woche), von den jungen Aussiedlern jedoch fast gar nicht.

Zusätzlich wurde in der LfM-Studie - ebenso wie in der JIM-Studie - gefragt, ob das Phänomen des „happy slapping“ als eines der Gefahrenpotenziale im Umgang mit dem Handy den jungen Aussiedlern bekannt ist bzw. ob sie dieses im Bekanntenkreis selbst erfahren haben. Davon gehört zu haben bzw. zu wissen, dass gewalthaltige Videos oder 
pornografische Bilder und Filme per Handy verschickt werden, signalisieren gleichermaßen rund vier Fünftel der Befragten der JIM-Studie und der jungen Aussiedler. Auch bei der Frage, ob man so etwas bereits im eigenen Freundes- oder Bekanntenkreis mitbekommen habe, unterscheiden sich die jungen Aussiedler und die 12- bis 19-Jährigen der Gesamtbevölkerung kaum (26\% vs. $29 \%$ ). Im Bereich finanzieller Risiken der Handynutzung haben die jungen Aussiedler bislang wenige negative Erfahrungen gemacht: Nur 11 Prozent von ihnen sind von ungewollten Vertragsabschlüssen und 8 Prozent von Kostenfallen durch teure Servicenummern betroffen gewesen.

\section{Digitale Spiele}

Wie die letzten Abschnitte gezeigt haben, ist das „digitale Spielen“ nicht zwangsweise mit spezifischen Spielgeräten verknüpft, sondern eine Funktion, die von allen digitalen Medien - online und offline, stationär und mobil - bereitgestellt wird. Im Rahmen der LfM-Studie wurde das Spielverhalten der jungen russischen Aussiedler in drei medialen Kontexten erfasst: mit Spielkonsolen (stationär und mobil), am PC (offline sowie im Internet) und mit dem Handy.

Betrachtet man diese Spielmöglichkeiten jeweils einzeln, sind die höchsten Nutzungswerte beim Spielen am PC festzustellen (insgesamt $29 \%$; online $21 \%$ ). An zweiter Stelle folgen die Spielkonsolen (22\%) und an letzter Stelle die Handy-Spiele (11\%). Fasst man alle digitalen Spielmöglichkeiten zusammen, kommt man in der Stichprobe der 12- bis 19-jährigen russischen Aussiedler zu 34 Prozent „Stammspielern“ (d. h. Personen, die mindestens an vier Tagen einer Woche spielen) bzw. zu 45 Prozent, die diese Spiele an mindestens drei Tagen pro Woche nutzen.

Angesichts der öffentlichen Debatten zu diesem Thema sind das relativ niedrige Werte. Sie entsprechen nicht nur den Durchschnittswerten, die im Rahmen der JIMStudie 2008 für das Spielverhalten aller gleichaltrigen Jugendlichen in Deutschland ermittelt worden sind. Den Computerspielern steht auch eine deutlich größere Gruppe junger Aussiedler gegenüber ( $55 \%$ ), für die diese Form der digitalen Unterhaltung keine zentrale Bedeutung hat. Im Übrigen unterscheiden sich die jungen russischen Aussiedler (zumindest nach ihren eigenen Angaben und anders, als auf der Basis einiger Studien [Eggert 2006, Fromme et al. 2000] zu erwarten wäre) nur wenig von den JIM-Befragten in ihren Vorlieben für bestimmte Spielarten (Action- und Kampfspiele vs. Strategiespiele).

\section{Statt einer Zusammenfassung: Mediennutzung und soziale Lebenswelten junger Migranten}

Betrachtet man die Daten der LfM-Studie zur Mediennutzung und Medienkompetenz von jungen, in Nordrhein-Westfalen lebenden Aussiedlern im Alter zwischen 12 und 19 Jahren aus komparatistischer Perspektive, vergleicht man sie also - wie im vorigen Kapitel geschehen - mit dem Medienumgang aller Jugendlichen dieser Altersgruppe in Deutschland, zeigen sich im Detail einige interessante Unterschiede. Insgesamt jedoch überwiegen bei Weitem die Gemeinsamkeiten einer aus diesem Blickwinkel anscheinend relativ homogenen "Jugendmedienkultur“. Das gilt ganz besonders für den Umgang mit den neuen digitalen Medien - d. h. genau für denjenigen Bereich, in dem der gesellschaftspolitische Diskurs am ehesten Probleme und Defizite im Medienverhalten junger Migranten vermutet. Zwar wird mit den hier präsentierten Daten nur die Oberflächenstruktur des Medienumgangs der jungen russischen Aussiedler skizziert. Dennoch ist 
festzuhalten, dass es zumindest auf dieser Ebene nur geringe Anzeichen für migrationstypische Verhaltensweisen und Einstellungen gibt.

Insofern stellt sich fast zwingend die Frage, ob innerhalb der Aussiedlerstichprobe migrationsunabhängige Einflussfaktoren auf den Medienumgang der befragten Jugendlichen identifiziert werden können. Wie wir an dieser Stelle nur noch kursorisch ausführen können, zeigt sich im Ergebnis solcher Analysen deutlich, dass auch in Migrantenpopulationen insbesondere das Lebensalter, das Geschlecht und die Schulbildung (als Indikatoren für spezifische Sub-Milieus bzw. Lebenslagen von Subgruppen ${ }^{29}$ ) - einen starken Einfluss auf die Quantität und Qualität des Medienumgangs haben.

Lebensalter. Die in diesem Beitrag betrachtete Altersgruppe ist zwar homogener als die Gesamtstichprobe junger russischer Aussiedler (12 bis 29 Jahre). Dennoch reicht sie von der späten Kindheit mit einer zum Teil noch recht engen Bindung an das Elternhaus bis zum unabhängigeren und stärker in Peer-Groups verankerten Jugendalter. Es ist daher nicht verwunderlich, dass man zu recht unterschiedlichen Befunden kommt, wenn man den Medienumgang der 12- bis 15-Jährigen mit dem der 16- bis 19-Jährigen kontrastiert.

Die Jüngeren sind noch ausgeprägter am Fernsehen orientiert als die Älteren und nicht so stark mit den neuen digitalen Medien befasst wie diese. Die digitale Welt ist vor allem für sie eine Welt des Spielens: ihre Nutzungswerte sind bei Spielkonsolen und Computerspielen (online und offline) doppelt so hoch wie die der über 15-Jährigen. Sie geben am wenigsten Geld für die Handynutzung aus und sind in der Handhabung der kommunikativen Handyfunktionen tatsächlich zurückhaltender als die Älteren. In der Nutzung der Unterhaltungsfunktionen des Handys als Musik- und Bildmedium unterscheiden sich die Altersgruppen jedoch nicht.

Die Älteren haben noch einen, wenn auch schwachen Zugang zu russischsprachigen Medien. Auf jeden Fall ist er stärker als bei den Jüngeren. Sie nutzen das Internet nicht nur generell häufiger, sondern vor allem in der Breite der verfügbaren Dienste und Funktionen sehr viel ausgeprägter als diese. Mit Ausnahme der Chats und Newsgroups betrifft das alle wichtigen Kommunikations- und Informationsdienste des Internets und im Unterhaltungsbereich vor allem die Verwendung des Internets als Musikmedium. Außerdem ist den Älteren die berufliche Relevanz eines kompetenten Umgangs mit den neuen EDV- und IT-Techniken sehr viel deutlicher bewusst als den Jüngeren.

Geschlecht. ${ }^{30}$ Die Mädchen in der Stichprobe der jungen russischen Aussiedler sind stärker auf die Nutzung von Massen- und Individualmedien fokussiert als die Jungen. Sie hören deutlich mehr Radio, aber auch bei Fernsehen, Internet und Handy sind ihre Nutzungswerte tendenziell höher. Eine zentrale Ausnahme betrifft das digitale Spielen, das - zumindest im Jugendalter - ganz offensichtlich eine männliche Domäne ist. Die Mädchen nutzen einerseits die Kommunikationsdienste des Internets (insbesondere EMail und Communities) sehr viel stärker als die Jungen. Andererseits schöpfen sie auch die schulisch und beruflich relevanten Informationsleistungen von Internet und PC intensiver aus. Tatsächlich macht ihnen das Lernen und Arbeiten am PC auch mehr Spaß als den Jungen. Und sie schätzen mehr als diese die sozialkommunikative Funktion des

29 Dass diese Indikatoren in unterschiedlichen Migrantenpopulationen für unterschiedliche Lebenswelten stehen, ist selbstverständlich und könnte z. B. durch einen Vergleich der jungen Mädchen in den beiden Migrantenstichproben der LfM-Studie belegt werden.

$30 \mathrm{Zu}$ den nachfolgenden Ausführungen ist allerdings zu beachten, dass es in der untersuchten Aussiedlerstichprobe einen relativ starken Zusammenhang zwischen Geschlecht und Schulstatus/Schulabschluss gibt: die Mädchen sind bei den Gymnasiasten, die männlichen Jugendlichen bei den Hauptschülern überrepräsentiert. 
Internets („Neue Leute kennen lernen“). Bei der Nutzung der Unterhaltungsfunktionen des Internets zeigt sich neben dem unterschiedlichen Spielverhalten eine weitere geschlechtsspezifische Differenz: Die Mädchen nutzen das Internet mehr, um Musik zu hören. Die Jungen wenden sich mehr den Filmen und Videos im Internet zu.

Schulbildung. Da in der Stichprobe der 12- bis 19-jährigen Aussiedler über 80 Prozent der Befragten noch die Schule besuchten, geht es hier mehr um den „Schülerstatus“ als um Schulabschlüsse. Im Vergleich der Mediennutzung der verschiedenen Schülergruppen zeigen sich - erwartungsgemäß - die größten Unterschiede zwischen Hauptschülern und Gymnasiasten. Erstaunlich ist jedoch die zumeist fast lineare Abstufung der einschlägigen Verhaltensweisen und Einstellungen über alle drei Schultypen hinweg.

Im Umgang mit Massenmedien unterscheiden sich die Schülergruppen nur geringfügig. Lediglich das Radiohören ist bei den Gymnasiasten und Realschülern stärker verbreitet als bei den Hauptschülern. Im Bereich der Individualmediennutzung ist dagegen ein klarer „Gap“ zwischen den Gymnasiasten einerseits und den restlichen Schülern andererseits festzustellen: Die Gymnasiasten sind die intensivsten PC-, Internet- und Handynutzer. Außerdem beschäftigen sie sich häufiger als die anderen Schüler auch mit russischen Medienangeboten. Am stärksten macht sich diese Differenz bei der Nutzung der Arbeitsmöglichkeiten am PC und der Informationsdienste des Internets für die Schule bemerkbar, stark aber auch bei der Nutzung der Kommunikationsfunktionen des Internets.

Umgekehrt wird im Blick auf den Umgang der Hauptschüler (und zum Teil auch der Realschüler) mit der IT-Technologie sehr schnell deutlich, dass der „Digital Divide“ innerhalb der Gruppe junger Aussiedler, und zwar insbesondere an deren Schulbiografien und Bildungschancen entlang, verläuft. Besonders aufschlussreich ist in diesem $\mathrm{Zu}-$ sammenhang, dass einerseits über 40 Prozent der Hauptschüler sagen, sie würden sich „gerne häufiger mit Computern beschäftigen“, und andererseits ebenfalls über 40 Prozent von ihnen dem Statement zustimmen „Meine Eltern halten nichts von Computern“. Schließlich sind sie auch bei denen überrepräsentiert, die sich in den Regeln der Internetnutzung unsicher sind (68\% Hauptschüler vs. $85 \%$ Gymnasiasten Zustimmung zu „Wenn ich das Internet nutze, weiß ich genau, was erlaubt ist und was nicht“).

Die zuletzt skizzierten Untersuchungsergebnisse müssten eigentlich, wie auch der Vergleich der Aussiedlerdaten mit Umfragen in der Gesamtbevölkerung, etwas „Normalität“ in die gesellschaftspolitische Erörterung und sozialwissenschaftliche Erforschung der Mediennutzung und Medienkompetenz von jungen Migranten in Deutschland bringen - und zugleich den Wert und die Notwendigkeit repräsentativer Forschung in diesem Feld unterstreichen.

\section{Literatur}

Baacke, Dieter (1973): Kommunikation und Kompetenz. München.

Baacke, Dieter (1999): Medienkompetenz als zentrales Operationsfeld von Projekten. In: Baacke, Dieter/Susanne Kornblum/Jürgen Lauffer/Lothar Mikos/Günter A. Thiele (Hrsg.): Handbuch Medien: Medienkompetenz. Modelle und Projekte. Bundeszentrale für politische Bildung. Bonn, S. 31-35.

Bundesministerium des Inneren (Hrsg.) (2008): Migrationsbericht des Bundesamtes für Migration und Flüchtlinge im Auftrag der Bundesregierung. Migrationsbericht 2007. Berlin, www.bmi.bund.de/cae/servlet/contentblob/297624/publicationFile/14809/migrationsbericht _2007.pdf;jsessionid=8FABF73FC503B616AC382F2D049EAD1C [22.07.2010] [Migrationsbericht 2007]. 
Bundesgesetzblatt Jahrgang 2007 Teil I Nr. 40: Gesetz über die Angelegenheiten der Vertriebenen und Flüchtlinge (Bundesvertriebenengesetz-BVFG), ausgegeben zu Bonn am 17. August 2007, S. 1902-1911.

Burkhardt, Wolfgang (2001): Förderung kindlicher Medienkompetenz durch die Eltern. Grundlagen, Konzepte, Zukunftsmodelle. Opladen (Schriftenreihe Medienforschung der Landesanstalt für Rundfunk Nordrhein-Westfalen; Bd. 40).

Der Beauftragte der Bundesregierung für Kultur und Medien (2008): Medien- und Kommunikationsbericht der Bundesregierung 2008. Berlin. [Medien- und Kommunikationsbericht 2008]

Dietz, Barbara (1997): Jugendliche Aussiedler. Ausreise, Aufnahme, Integration. Berlin (Schriftenreihe Aussiedlerintegration; Bd. 7).

Dietz, Barbara/Heike Roll (1998): Jugendliche Aussiedler - Porträt einer Zuwanderergeneration. Frankfurt/Main, New York.

Düvel, Caroline (2009): Lokal - translokal - digital: Kommunikative Mehrfachvernetzung und die Aneignung digitaler Medienumgebungen in der russischen Diaspora. In: Hunger, Uwe/Kathrin Kissau (Hrsg.): Internet und Migration. Theoretische Zugänge und empirische Befunde. Wiesbaden, S. 257-277.

Eckhardt, Josef (2000): Mediennutzungsverhalten von Ausländern in Deutschland. In: Schatz, Heribert/Christina Holtz-Bacha/Jörg-Uwe Nieland (Hrsg.): Migranten und Medien. Neue Herausforderungen an die Integrationsfunktion von Presse und Rundfunk. Wiesbaden, S. 265-271.

Eggert, Susanne (2006): Von „mail.ru“ bis „ProSieben“-Zur Medienaneignung Heranwachsender aus der ehemaligen Sowjetunion. In: Treibel, Annette/Maja S. Maier/Sven Kommer/Manuela Welzel (Hrsg.): Gender medienkompetent. Medienbildung in einer heterogenen Gesellschaft. Wiesbaden, S. 235-255.

Elias, Nelly/Dafna Lemish (2008): Medien, Migrationserfahrung und Adoleszenz. Die Rolle von Fernsehen und Internet für russischstämmige 6- bis 18-Jährige in Deutschland und Israel. In: Televizion, Heft 21/1, S. 24-28.

Fromme, Johannes/Norbert Meder/Nikolaus Vollmer (2000): Computerspiele in der Kinderkultur. Opladen (Reihe Virtuelle Welten; Bd. 1).

Geißler, Rainer/Sonja Weber-Menges (2008): Migrantenkinder im Bildungssystem: doppelt benachteiligt. In: APuZ (Aus Politik und Zeitgeschichte), Heft 49, S. 14-22.

Geißler, Rainer/Sonja Weber-Menges (2009): Media Reception and Ideas on Media Integration among Turkish, Italian and Russo-German Migrants in Germany. In: Geißler, Rainer/Horst Pöttker (Eds.): Media - Migration - Integration. European and North American Perspectives. Bielefeld, S. 27-43.

Hepp, Andreas/Laura Suna/Stefan Welling (2009): Kommunikative Vernetzung, Medienrepertoires und kulturelle Zugehörigkeit: Die Aneignung digitaler Medien in der polnischen und russischen Diaspora. In: Hunger, Uwe/Kathrin Kissau (Hrsg.): Internet und Migration. Theoretische Zugänge und empirische Befunde. Wiesbaden, S. 173-197.

Humpert, Andreas/Klaus Schneiderheinze (2000): Stichprobenziehung für telefonische Zuwandererumfragen - Einsatzmöglichkeiten der Namenforschung (Onomastik). In: ZUMA-Nachrichten, Heft 47, S. 36-63.

Ingenhorst, Heinz (1997): Die Russlanddeutschen. Aussiedler zwischen Tradition und Moderne. Frankfurt/Main.

Initiative D21 e. V. (Hrsg.) (2008): Internetnutzung und Migrationshintergrund in Deutschland. Eine Sonderauswertung zum (N)ONLINER Atlas 2008. ohne Ort, http://old.initiatived21.de/ fileadmin/files/08_NOA/NOA_Migration.pdf [23.07.2010].

Ipsen-Peitzmeier, Sabine/Markus Kaiser (Hrsg.) (2006): Zuhause fremd - Russlanddeutsche zwischen Russland und Deutschland. Bielefeld.

Ivanova, Ana (2009): Kommunikationsverhalten, Identität und Integration von russlanddeutschen Aussiedlern (1999). Eine Sekundäranalyse. Berlin: Freie Universität Berlin (unveröff. Magisterarbeit).

Kissau, Kathrin (2008): Das Integrationspotential des Internet für Migranten. Wiesbaden.

Kissau, Kathrin/Uwe Hunger (2009): Politische Sphären von Migranten im Internet. Neue Chancen im „Long Tail“ der Politik. München. 
Kubicek, Herbert/Stefan Welling (2000): Vor einer digitalen Spaltung in Deutschland? Annäherung an ein verdecktes Problem von wirtschafts- und gesellschaftspolitischer Brisanz. In: Medien \& Kommunikationswissenschaft, Jg. 48, Heft 4, S. 497-516.

Luca, Renate/Stefan Aufenanger (2007): Geschlechtersensible Medienkompetenzförderung. Mediennutzung und Medienkompetenz von Mädchen und Jungen sowie medienpädagogische Handlungsmöglichkeiten. Berlin (Schriftenreihe Medienforschung der LfM Nordrhein-Westfalen; Bd. 58).

Medienpädagogischer Forschungsverbund Südwest (mpfs) (Hrsg.) (2007): JIM 2007. Jugend, Information, (Multi-)Media. Basisstudie zum Medienumgang 12- bis 19-Jähriger in Deutschland. Stuttgart.

Medienpädagogischer Forschungsverbund Südwest (mpfs) (Hrsg.) (2008): JIM 2008. Jugend, Information, (Multi-)Media. Basisstudie zum Medienumgang 12- bis 19-Jähriger in Deutschland. Stuttgart.

Medienpädagogischer Forschungsverbund Südwest (mpfs) (Hrsg.) (2009a): KIM-Studie 2008. Kinder und Medien, Computer und Internet. Basisuntersuchung zum Medienumgang 6- bis 13Jähriger in Deutschland. Stuttgart.

Medienpädagogischer Forschungsverbund Südwest (mpfs) (Hrsg.) (2009b): JIM 2009. Jugend, Information, (Multi-)Media. Basisstudie zum Medienumgang 12- bis 19-Jähriger in Deutschland. Stuttgart.

Niesyto, Horst (2008): Die soziale Frage in Medienforschung und Medienpädagogik. In: Dokumentation des Fachkongresses „Soziale Ungleichheit, Medienpädagogik, Partizipation“ 17./18.10.2008, Bonn, JFF - Institut für Medienpädagogik/Pädagogische Hochschule Ludwigsburg, Teil 2, S. 6-24, www.jff.de/dateien/Tagungsdoku_SozU_2ter_Teil.pdf [26.07.2010].

Niesyto, Horst (2009): Digitale Medien, soziale Benachteiligung und soziale Distinktion. In: MedienPädagogik. Zeitschrift für Theorie und Praxis der Medienbildung. Themenheft Nr. 17: Medien und soziokulturelle Unterschiede, www.medienpaed.com/17/niesyto0906.pdf [26.07.2010].

Oehmichen, Ekkehardt (2007): Radionutzung von Migranten. Ergebnisse der ARD/ZDF-Studie „Migranten und Medien“. In: Media Perspektiven, Heft 9, S. 452-460.

Pfetsch, Barbara/Hans-Jürgen Weiß (2000): Die kritische Rolle der Massenmedien bei der Integration sozialer Minderheiten. Anmerkungen aus einem deutsch-israelischen Forschungsprojekt. In: Schatz, Heribert/Christina Holtz-Bacha/Jörg-Uwe Nieland (Hrsg.): Migranten und Medien. Neue Herausforderungen an die Integrationsfunktion von Presse und Rundfunk. Wiesbaden, S. 116-126.

Pfetsch, Barbara/Joachim Trebbe (2003): Mass Media Use and Social Integration of German-Russian Immigrants in Germany. San Diego (Paper submitted to the 53rd Annual Conference of the International Communication Association, May 23-27, 2003, San Diego).

Ridder, Christa-Maria/Bernhard Engel (2005): Massenkommunikation 2005: Images und Funktionen der Massenmedien im Vergleich. In: Media Perspektiven, Heft 9, S. 422-448.

Schäfer, Heiner (2002): „Junge Russen“ in Deutschland - Aussiedler verloren zwischen Herkunft und Zukunft? In: Arbeitsstelle Kinder- und Jugendkriminalitätsprävention (Hrsg.): Die mitgenommene Generation. Aussiedlerjugendliche - eine pädagogische Herausforderung für die Kriminalitätsprävention. München, S. 12-67.

Schneider, Jan (2005): Die Geschichte der Russlanddeutschen, www.bpb.de/themen/AA1Q8R, 4,0,Die_Geschichte_der_Russlanddeutschen.html\#art4 [26.07.2010].

Seifert, Wolfgang (2008): Aussiedlerinnen und Aussiedler - neue Erfassungsmöglichkeiten und sozioökonomisches Profil. In: Landesamt für Datenverarbeitung und Statistik Nordrhein-Westfalen (Hrsg.): Statistische Analysen und Studien Nordrhein-Westfalen. Band 53. Düsseldorf, S.11-23, www.it.nrw.de/statistik/analysen/stat_studien/2008/band_53/wl_seifert_55.html [26.07.2010].

Silbereisen, Rainer K./Ernst-Dieter Lantermann/Eva Schmitt-Rodermund (Hrsg.) (1999a): Aussiedler in Deutschland. Akkulturation von Persönlichkeit und Verhalten. Opladen.

Silbereisen, Rainer K./Eva Schmitt-Rodermund/Ernst-Dieter Lantermann (1999b): Hintergrund, theoretische Perspektiven, Anlage und Themen der Aussiedlerstudie. In: Silbereisen, Rainer K./ 
Ernst-Dieter Lantermann/Eva Schmitt-Rodermund (Hrsg.): Aussiedler in Deutschland. Akkulturation von Persönlichkeit und Verhalten. Opladen. S. 13-45.

Simon, Erk (2007): Migranten und Medien 2007: Zielsetzung, Konzeption und Basisdaten einer repräsentativen Studie der ARD/ZDF-Medienkommission. In: Media Perspektiven, Heft 9, S. 426-435.

Six, Ulrike/Roland Gimmler (2007): Die Förderung von Medienkompetenz im Kindergarten. Eine empirische Studie zu Bedingungen und Handlungsformen der Medienerziehung. Berlin (Schriftenreihe Medienforschung der Landesanstalt für Medien Nordrhein-Westfalen; Bd. 57).

Statistisches Bundesamt (2009): Bevölkerung und Erwerbstätigkeit. Bevölkerung mit Migrationshintergrund - Ergebnisse des Mikrozensus 2007. Wiesbaden. www-ec.destatis.de/csp/shop/ sfg/bpm.html.cms.cBroker.cls? cmspath=struktur,vollanzeige.csp\&ID=1023128 [26.07.2010].

Strobl, Rainer (2006): Chancen und Probleme der Integration junger Aussiedler aus der früheren Sowjetunion. In: Ipsen-Peitzmeier, Sabine/Markus Kaiser (Hrsg.): Zuhause fremd - Russlanddeutsche zwischen Russland und Deutschland. Bielefeld. S. 87-107.

Theunert, Helga (1999): Medienkompetenz: Eine pädagogisch und altersspezifisch zu fassende Handlungsdimension. In: Schell, Fred/Elke Stolzenburg/Helga Theunert (Hrsg.): Medienkompetenz: Grundlagen und pädagogisches Handeln. München, S. 50-59.

Theunert, Helga (2008): Jugendmedienschutz und Medienkompetenz: Kongruenz, Koexistenz, Konkurrenz. In: Dörken-Kucharz, Thomas (Hrsg.): Medienkompetenz. Zauberwort oder Leerformel des Jugendmedienschutzes? Baden-Baden, S. 35-46.

Trebbe, Joachim (2009): Ethnische Minderheiten, Massenmedien und Integration. Eine Untersuchung zu massenmedialer Repräsentation und Medienwirkungen. Wiesbaden (Habilitationsschrift am Fachbereich Politik und Sozialwissenschaften der Freien Universität Berlin).

Trebbe, Joachim/Annett Heft/Hans-Jürgen Weiß (2010): Mediennutzung junger Menschen mit Migrationshintergrund. Umfragen und Gruppendiskussionen mit Personen türkischer Herkunft und russischen Aussiedlern im Alter zwischen 12 und 29 Jahren in Nordrhein-Westfalen. Berlin (Schriftenreihe Medienforschung der LfM Nordrhein-Westfalen; Bd. 63).

Treibel, Annette (2006): Medienkompetenzen an der Hauptschule. Zur Relevanz von Migration, Gender und Individualisierung bei russlanddeutschen und türkischstämmigen Jugendlichen. In: Treibel, Annette/Maja S. Maier/Sven Kommer/Manuela Welzel (Hrsg.): Gender medienkompetent. Medienbildung in einer heterogenen Gesellschaft. Wiesbaden, S. 209-233.

Treumann, Klaus Peter/Dorothee M. Meister/Uwe Sander/Eckhard Burkatzki/Jörg Hagedorn/ Manuela Kämmerer/Mareike Strotmann/Claudia Wegener (2007): Medienhandeln Jugendlicher. Mediennutzung und Medienkompetenz. Bielefelder Medienkompetenzmodell. Wiesbaden.

Van Dijk, Jan A. G. M. (2005): The Deepening Divide. Inequality in the Information Society. Thousand Oaks, London, New Delhi.

Vogelgesang, Waldemar (2008): Jugendliche Aussiedler. Zwischen Entwurzelung, Ausgrenzung und Integration. Weinheim und München.

Wagner, Ulrike (Hrsg.) (2008): Medienhandeln in Hauptschulmilieus. Mediale Interaktion und Produktion als Bildungsressource. München.

Windgasse, Thomas (2007): Die Radionutzung von Migranten im Kontext anderer Medien. Ergebnisse einer Repräsentativstudie in Nordrhein-Westfalen. In: Media Perspektiven, Heft 3, S. 153-161.

Zillien, Nicole (2006): Digitale Ungleichheit. Neue Technologien und alte Ungleichheiten in der Informations- und Wissensgesellschaft. Wiesbaden. 